\title{
Measuring Uncertainty in International Relations: Heteroskedastic Strategic Models
}

\section{Citation}

Bas, M. A. 2012. “Measuring Uncertainty in International Relations: Heteroskedastic Strategic Models." Conflict Management and Peace Science 29 (5) (November 1): 490-520. doi:10.1177/0738894212449088.

\section{Published Version}

doi:10.1177/0738894212449088

\section{Permanent link}

http://nrs.harvard.edu/urn-3:HUL.InstRepos:22547791

\section{Terms of Use}

This article was downloaded from Harvard University's DASH repository, and is made available under the terms and conditions applicable to Open Access Policy Articles, as set forth at http:// nrs.harvard.edu/urn-3:HUL.InstRepos:dash.current.terms-of-use\#OAP

\section{Share Your Story}

The Harvard community has made this article openly available.

Please share how this access benefits you. Submit a story.

\section{Accessibility}




\title{
Measuring Uncertainty in International Relations: Heteroskedastic Strategic Models
}

\author{
Muhammet A. Bas * \\ Department of Government \\ Harvard University \\ 1737 Cambridge Street \\ Cambridge, MA 02138 \\ Email: mbas@gov.harvard.edu
}

\begin{abstract}
Keywords:
heteroskedasticity, strategic interaction, uncertainty in IR private information, bounded rationality
\end{abstract}

\begin{abstract}
Actor-level variations in the amounts of uncertainty has been widely ignored in the growing literature on statistical models of strategic interaction in international relations. In this paper, I provide a tool for testing theories about the level of uncertainty in strategic interactions. I show that ignoring potential variations in levels of uncertainty across different cases can be a source of bias for empirical analyses. I propose a method to incorporate this form of heteroskedasticity into existing estimators and show that this method can improve inferences. With a series of Monte Carlo experiments, I evaluate the magnitude and the severity of the bias and inconsistency in estimators that ignore heteroskedasticity. More importantly, the tools developed in this paper have many interesting substantive application areas. Examples considered include measuring speculators' suboptimal behavior tendencies in international currency crises; and capturing varying levels of signaling and Bayesian updating behavior in the recent strategic models of signaling.
\end{abstract}

*This paper was previously presented at the 2010 Annual Meeting of the Midwest Political Science Association. I would like to thank Curtis Signorino, Elena McLean, Taehee Whang and David Carter for valuable comments. I also thank the editor and the anonymous reviewers. All errors remain my own. 


\section{Introduction}

In recent years, increased attention has been given in the international relations literature to testing hypotheses about strategic interaction (Fearon, 1994b; Smith, 1999; Signorino, 1999; Schultz, 1999; Leblang, 2003; Signorino and Tarar, 2006; Bas, Signorino and Walker, 2008; McLean and Whang, 2010). More recently, scholars have focused on making sure that empirical models used to test theories about strategic interaction are compatible with these theories. Alternative estimators are developed depending on the form of the underlying strategic interaction, ranging from simple 2-by-2 discrete choice dynamic games to games of signaling, and dynamic continuous choice games (Signorino, 1999; Lewis and Schultz, 2003; Esarey, Mukherjee and Moore, 2008; Ramsay and Signorino, 2009; Whang, 2010). Whether the topic is deterrence (Signorino and Tarar, 2006), economic sanctions (Whang, 2010), or international currency crises (Leblang, 2003), the use of these estimators made it possible for the empirical estimation to follow closely and remain compatible with the underlying theoretical model that involves strategic interaction.

One major advantage of these estimators - strategic models - is that they enable testing non-monotonic hypotheses out of a theoretical model about the effects of covariates of interest on strategic actor behavior. Such hypotheses are hard to test with traditional logit or probit regression models. ${ }^{1}$ Signorino and Yilmaz (2003) show that using logit or probit regression models to capture strategic interaction results in functional form misspecification akin to an omitted variable bias.

Unlike in linear regression or probit, the error terms in strategic models are not just nuisance parameters. They are usually theoretically motivated, and it is this feature that makes statistically estimating a strategic model possible by providing the transition from a

\footnotetext{
${ }^{1}$ There is a debate in the literature about the usefulness of logit or probit in testing non-monotonic hypotheses arising from strategic interaction. See Carrubba, Yuen and Zorn (2007a), Signorino (2007) and Carrubba, Yuen and Zorn (2007b) for this debate.
} 
game theoretical model to a statistical model. One theoretical source of uncertainty proposed, for instance, is the existence of boundedly rational actors that randomly deviate from rational behavior in an unpredictable manner during game play (Signorino, 2003). ${ }^{2}$ More frequently, private information that actors have about their payoffs has been used as a source of uncertainty that converts game theoretical models into statistical models that can be estimated (Signorino, 2003; Lewis and Schultz, 2003; Whang, 2010). Signorino (2003) shows that what the analyst chooses as the source of uncertainty in his or her statistical model is important because each type of uncertainty results in different probability models and the misspecification of the error structure can result in bias and inconsistency.

Regardless of the type of uncertainty, one common assumption in the existing statistical strategic models is that the uncertainty is homoskedastic. If the source of uncertainty is agents' bounded rationality, homoskedasticity means that all the actors in the data set have the same tendency to deviate from rational behavior, or that there is only one distribution with constant mean and variance that represents the actors' and the analyst's prior belief about a given actor's deviations from rational behavior. Similarly, if the source of uncertainty is private information, homoskedasticity implies that all the actors in the sample have the same amount of private information about their payoffs.

Should researchers have any reasons to suspect heteroskedasticity in a strategic model in international relations? Uncertainty is at the heart of strategic interaction, and accordingly it plays an important role in theories of strategic interaction in international relations. ${ }^{3}$ Many theories predict that levels of uncertainty vary across different actors or in different interactions (Fearon, 1994a; Schultz, 1998). Therefore, regardless of whether one is dealing with bounded rationality or private information as the theoretical source of uncertainty, the

\footnotetext{
${ }^{2}$ The same assumption about actor rationality exists in McKelvey and Palfrey's 1995; 1998 Quantal Response Equilibrium.

${ }^{3}$ For instance, in an influential article, Fearon (1995) argues that uncertainty is one of the main reasons why rational states fight instead of reaching mutually preferable peaceful bargaining solutions.
} 
answer to the above question is likely to be yes. ${ }^{4}$ For instance, in making expected utility comparisons, players might have differing tendencies to deviate from optimality. Bounded rationality is an individual characteristic, and as such the tendency to err is highly likely to vary across different actors in a given data set. Even with the same actor, this tendency might vary depending on time, the environment, or the specific actors faced in a strategic interaction. Assuming that everyone has the same likelihood and tendency to make miscalculations might be too restrictive. Similarly, in a private information specification, the severity of information asymmetry can change depending on actors' characteristics or the strategic environment. In the international conflict literature, for instance, there is a big debate about the effect of regime type on the amount of private information states have in international conflict (Fearon, 1994a; Schultz, 1998, 1999, 2001). By limiting their focus to homoskedastic models, empirical international relations scholars cannot incorporate these possibilities into their research.

What are the consequences of disregarding heteroskedasticity? It has been widely known that heteroskedasticity is only an efficiency problem in linear regression, and one's estimates remain unbiased and consistent even if he or she does not model heteroskedasticity (Greene, 2003). This result is not generalizable, however, to nonlinear regression models with discrete dependent variables such as logit or probit (Yatchew and Griliches, 1985). As strategic models can be regarded as multi-equation systems of probit and logit models, when not correctly modeled, heteroskedasticity might cause biased and inconsistent estimates in strategic models as well. In other words, when we have theoretical expectations that each player in a strategic model has a different tendency to make suboptimal choices, or different players possess different amounts of private information in a strategic situation, estimating conven-

\footnotetext{
${ }^{4} \mathrm{~A}$ quick look at the existing empirical analyses in the literature that employ strategic models supports this statement. Most of these analyses use panel data, combining many actors that are observed over a long period of time. Leblang (2003), for instance, uses a data set consisting of monthly observations of 90 developing economies from January 1985 to December 1998. It is highly likely that the levels of uncertainty will show variation in such diverse samples.
} 
tional strategic probit or logit models with fixed homoskedastic error variances may result in bias. ${ }^{5}$

This paper aims to contribute to the burgeoning literature on strategic models and complement the existing estimators in three ways: first, I propose a method, heteroskedastic strategic probit (HSP), that helps capture heteroskedasticity in strategic models and correct the potential bias and inconsistency when the data generating process violates the homoskedasticity assumption. ${ }^{6}$ I demonstrate that HSP should be preferred over homoskedastic SP when heteroskedasticity is suspected, except in very small samples, due to the relative inefficiency and potential fragility of HSP in such samples. Secondly, and more importantly, I show that HSP makes possible addressing many substantively interesting research questions by providing scholars with an opportunity to measure different types of actor-level uncertainty that exist in the strategic interactions they analyze. Finally, I show that the proposed method can have better identification properties in the sense that, under certain conditions, it enables simultaneous estimation of multiple theoretical sources of uncertainty in a given model, which is not possible to accomplish with existing homoskedastic strategic models.

Scholars have previously noted a lack of attention given to theorizing the variance parameter - the "second moment" - in the empirical international relations literature (Braumoeller, 2006). They emphasized the importance and the potential value of substantively interpreting heteroskedasticity instead of treating it just a nuisance (Downs and Rocke, 1979). Due to the important role uncertainty plays in strategic interaction, it is even more important -

\footnotetext{
${ }^{5}$ The potential problems associated with a constant variance assumption have been acknowledged in the recent literature on strategic models. In comparing different models of strategic choice, Wand (2006) notes that different choices for the common constant variance parameter in strategic models result in different model predictions. Thus, if the data violates common variance assumption, one would expect that the failure to take this into account in the statistical model would bias inferences.

${ }^{6}$ I use the label strategic probit (SP) to refer to strategic estimators with Normally distributed uncertainty structure, as in Signorino (2003).
} 
and substantively more promising - to develop and test theories about variance in strategic models. Thus, the HSP developed in this paper will hopefully prove to be very useful to empirical scholars of international relations.

The paper proceeds as follows. In the next section, I derive the HSP estimator for a simple 2-player discrete choice game. Then, using Monte Carlo simulations, I analyze the bias in existing strategic models that do not model heteroskedasticity; investigate the potential effects of using a heteroskedastic estimator when heteroskedasticity is not present; and examine the properties of a hybrid heteroskedastic model that incorporates multiple sources of uncertainty. Finally, I discuss two application areas for HSP models. I first replicate Leblang's (2003) analysis of speculative currency attacks using the HSP estimator, and then discuss the potential extension of the technique to the recent statistical strategic models of signaling and Bayesian updating.

\section{Heteroskedastic Strategic Probit}

Signorino (2003) proposes two distinct theoretical sources of uncertainty in statistical strategic models. One source of uncertainty is agent error. With the agent error specification, the analyst assumes that players are boundedly rational, or they tend to misperceive each other's utilities, or they make errors in implementing their actions. A second source of uncertainty is private information. In this specification, a player possesses private information about his payoff from a particular outcome. It is assumed that only the player observes his true utility from an outcome; his opponent and the analyst regard it as random and can only know its

distribution. In sum, "agent error allows for nonrational behavior or 'accidents', whereas private information allows for rational behavior and incomplete information" (Signorino, 2003). 
[Figure 1 about here]

Figure 1 represents these two sources of uncertainty graphically in the context of a simple game theoretical model of international conflict between two states, denoted as Player $1\left(P_{1}\right)$ and Player $2\left(P_{2}\right) \cdot{ }^{7}$ In this sequential model, $P_{1}$ moves first and chooses between attacking $P_{2}$ or not. If $P_{1}$ does not attack, Status $Q u o$ is the outcome. If $P_{1}$ attacks, then $P_{2}$ decides to resist or capitulate. In the first case, the outcome is War. In the latter case, the outcome of the game is Capitulation. Players have utilities associated with each outcome, and expected utilities associated with each action that are derived based on the outcome utilities. Figure 1(A) depicts an agent error specification: error is associated with Player 1's comparison of expected utilities of deciding to attack or not. That is, $P_{1}$ makes an implementation error or miscalculation while comparing the choices of attacking and not attacking. The random component $\alpha_{i j}$ represents the suboptimal behavior by $P_{i}$ for choosing the action $j$ that cannot be predicted by the opponent or the analyst. Similarly, Figure 1(B) presents a private information specification, in which the random components $\varepsilon_{i j}$ are attached to $P_{i}$ 's utilities, representing Player $i$ 's private information about his or her payoff from outcome j. These components cannot be observed directly by the opponent or the analyst, who only have distributional information about the utility values.

The HSP model that I describe in this section shares an important feature with the heteroskedastic probit model developed by Alvarez and Brehm $(1995,2002)$ to analyze the heterogeneity in survey responses to abortion policy issues. In Alvarez and Brehm's heteroskedastic probit model, the error standard deviation $\sigma_{i}$ is assumed to vary across observations. In particular, $\sigma_{i}$ is linked to regressors through a parametric functional form. This

\footnotetext{
${ }^{7}$ Although I introduce the heteroskedastic model with very a simple two-player sequential game to keep the presentation simple in this paper, it is easy to incorporate the technique to more elaborate games such as the International Interaction Game analyzed in Bueno de Mesquita and Lalman (1992) and Signorino (1999). Also, heteroskedasticity can be incorporated into more complex games with signalling and Bayesian updating (Lewis and Schultz, 2003; Whang, 2010). Later in the paper, I discuss the implications of ignoring heteroskedasticity and the substantive benefits from modeling it in such games.
} 
intuition will also underlie the HSP model presented below. Harvey (1976)'s multiplicative heteroskedasticity specification will be used throughout the paper to model heteroskedasticity, in which the error standard deviation takes the following form:

$$
\sigma=\exp (\mathbf{Z} \alpha)
$$

where $\mathbf{Z}$ is the matrix of regressors that are assumed to affect the variance of the error term, and $\alpha$ is the coefficient vector for these regressors. ${ }^{8}$

I now derive the HSP outcome probabilities and the log-likelihood function for the agent error model presented in Figure $1(\mathrm{~A}){ }^{9}$

\subsection{Heteroskedastic SP with Agent Error Specification}

The true utility for Player $i$ from outcome $Y_{j}, j \in\{1,2,3\}$ is $U_{i}\left(Y_{j}\right)$, and both players can observe this. Similarly, the expected utility calculated - potentially with error - by Player $i$ for action $\mathrm{a}_{\mathrm{k}}, \mathrm{k} \in\{1,2,3,4\}$ is $\mathbb{E} \mathbb{U}_{i}^{*}\left(a_{k}\right)$. The analyst and the opponent cannot observe the exact deviation from optimality involved in the calculation, but they possess distributional information about agent error terms $\alpha_{i k}$, which are assumed to follow a Normal distribution with zero mean and variance $\nu_{\mathrm{ik}}^{2}$, denoted $\mathrm{N}\left(0, \nu_{\mathrm{ik}}^{2}\right)$. Thus, the analyst and the opponent can calculate the expected value of the expected utilities with miscalculations averaged out, which is $\mathbb{E} \mathbb{U}_{i}\left(a_{k}\right)$.

In order to derive the choice probabilities, we need to work backwards up the game tree as in the homoskedastic version presented in Signorino (2003). Denote Player 1's probability of choosing action $a_{2}$ as $p_{1}^{A E}$, and Player 2's probability of choosing $a_{4}$ as $p_{2}^{A E}$. Player 2

\footnotetext{
${ }^{8}$ In a homoskedastic SP model, in contrast, depending on the interpretation, variance of the uncertainty term is either assumed to be a known constant, usually 1, or the coefficients are estimated and reported to a scale of the unknown standard deviation, because of identification restrictions.

${ }^{9}$ The private information version can be derived similarly, which is presented in the Appendix.
} 
chooses $a_{4}$ over $a_{3}$ if $\mathbb{E} \mathbb{U}_{2}^{*}\left(a_{4}\right) \geq \mathbb{E} \mathbb{U}_{2}^{*}\left(a_{3}\right)$. Thus,

$$
\begin{aligned}
p_{2}^{A E} & =\operatorname{Pr}\left(\mathbb{E} \mathbb{U}_{2}^{*}\left(a_{4}\right) \geq \mathbb{E U}_{2}^{*}\left(a_{3}\right)\right) \\
& =\operatorname{Pr}\left(U_{2}\left(Y_{3}\right)+\alpha_{24} \geq U_{2}\left(Y_{2}\right)+\alpha_{23}\right) \\
& =\Phi\left(\frac{U_{2}\left(Y_{3}\right)-U_{2}\left(Y_{2}\right)}{\sqrt{\nu_{23}^{2}+\nu_{24}^{2}}}\right)
\end{aligned}
$$

Deriving Player 1's choice probability can be achieved in a similar way:

$$
\begin{aligned}
p_{1}^{A E} & =\operatorname{Pr}\left(\mathbb{E} \mathbb{U}_{1}^{*}\left(a_{2}\right) \geq \mathbb{E}_{1}^{*}\left(a_{1}\right)\right) \\
& =\operatorname{Pr}\left(\left(1-p_{2}^{A E}\right)\left(U_{1}\left(Y_{2}\right)\right)+p_{2}^{A E}\left(U_{1}\left(Y_{3}\right)\right)+\alpha_{12} \geq U_{1}\left(Y_{1}\right)+\alpha_{11}\right) \\
& =\Phi\left(\frac{\left(1-p_{2}^{A E}\right) U_{1}\left(Y_{2}\right)+p_{2}^{A E} U_{1}\left(Y_{3}\right)-U_{1}\left(Y_{1}\right)}{\sqrt{\nu_{11}^{2}+\nu_{12}^{2}}}\right)
\end{aligned}
$$

Based on these action probabilities, the probability of reaching a particular outcome in the strategic model depicted in Figure 1 can be calculated:

$$
\begin{aligned}
\operatorname{Pr}^{A E}\left(Y_{1}\right) & =1-p_{1}^{A E} \\
\operatorname{Pr}^{A E}\left(Y_{2}\right) & =p_{1}^{A E}\left(1-p_{2}^{A E}\right) \\
\operatorname{Pr}^{A E}\left(Y_{3}\right) & =p_{1}^{A E} p_{2}^{A E}
\end{aligned}
$$

How do we specify the heteroskedastic agent error variance in the above model? The variance of the stochastic $\alpha_{\mathrm{ij}}$ terms represent the players' tendency to miscalculate and deviate from the optimal action. $\alpha_{\mathrm{ij}}$ terms are distributed normally with zero mean and variance $\nu_{\mathrm{ij}}^{2}$. The standard deviations $\nu_{i j}$ vary across different observations as functions of regressors $\mathbf{Z}$ :

$$
\nu_{\mathrm{ij}}=\exp (\gamma \mathbf{Z})
$$

This error specification is in the multiplicative form as discussed in Harvey (1976). There is no constant term in the error specification because of identification restrictions. ${ }^{10}$ When

\footnotetext{
${ }^{10}$ This is akin to assuming that the variance parameter is equal to one in a homoskedastic probit model. An alternative sometimes considered in the literature is not making this assumption, and instead estimating coefficients scaled by the square root of the unknown constant variance parameter. Similarly, an unknown constant term can be added to the heteroskedastic error structure, which would imply that the coefficient
} 
$\gamma=0$, the variance term becomes constant across observations, and the heteroskedastic model reduces to a homoskedastic SP model. For $\gamma \neq 0$, this specification allows each observation (or actor) to have a potentially different error variance. In other words, if agents are state leaders, each leader potentially has a different tendency to deviate from optimal expected utility calculation, captured by the variance of the agent error term.

The log-likelihood function for the HSP estimator with agent error specification is as follows:

$$
L^{A E}=\sum_{n=1}^{N} \sum_{j=1}^{3} I_{n j} \ln \left(\operatorname{Pr}^{A E}\left(Y_{n j}\right)\right)
$$

where $I_{n j}$ is the indicator function that takes the value 1 when the outcome is $j$ in observation $n$ and zero otherwise. $\operatorname{Pr}^{A E}\left(Y_{n j}\right)$ is the outcome probability for outcome $j$ in a given observation $n$.

This model can be estimated very easily in common statistical software like Stata, R, or Gauss. An example Stata code is included in the Appendix that shows how to define the log-likelihood function and run the estimation procedure.

\subsection{A Hybrid Model with Two Sources of Uncertainty}

Agent error and private information as two sources of uncertainty need not be mutually exclusive in a given strategic interaction. In fact, one can easily argue that, as two distinct theoretical concepts, both sources of uncertainty are present in a strategic situation. We can have players possessing private information about their payoffs, and at the same time making miscalculations or implementation errors in their choices. In this case, a researcher would need to be able to estimate the two sources simultaneously in a SP model. When the errors are assumed to have constant variance, as in a homoskedastic model, a hybrid model of this estimates in a player's utilities are estimated to a scale. 
sort cannot be estimated, simply because the model cannot identify and isolate both error variances. On the other hand, when certain identification restrictions are satisfied, these two sources of uncertainty can be incorporated into a single HSP model. In particular, when the two sources of uncertainty are linked to mutually exclusive sets of regressors, a hybrid model that incorporates both the agent error and private information specifications can be estimated. For instance, in a crisis interaction model in international relations, based on theoretical expectations from the literature, actor characteristics such as age, or experience can be used to model bounded rationality, while regressors at the interaction or system level such as system polarity, the distance between the two countries in conflict, or regime type similarity, can be used to model private information variance. When shared factors are hypothesized to affect the two error variances for a given player, however, a hybrid heteroskedastic model will no longer be identified, as two separate coefficients for a regressor that appears in both uncertainty variances cannot be estimated simultaneously. ${ }^{11}$

One obvious question that arises is what would happen when the underlying model is a hybrid heteroskedastic model that incorporates both types of uncertainty, but the analyst estimates either an agent error or private information specification? Similarly, if a hybrid model cannot be estimated due to identification problems stemming from shared regressors, would a private information or agent error heteroskedastic model be a close enough approximation? One of the Monte Carlo experiments I conduct in the next section will address these issues.

\footnotetext{
${ }^{11}$ The derivations of the hybrid heteroskedastic probit choice probabilities are a simple extension of the derivations for the agent error and private information specifications, and therefore not presented here. They are available from the author upon request.
} 


\section{Monte Carlo Analyses}

In this section, I conduct three sets of Monte Carlo analyses to assess the statistical properties of various HSP models based on the simple game structure in Figure $1 .^{12}$ In the first set of simulations, I evaluate the bias in a homoskedastic SP model when the data generating process is heteroskedastic. Then, in the second set, I look at the potential bias and inefficiency in fitting a HSP model when there is no heteroskedasticity in the data generating process. The last set of results is presented to demonstrate the better identification properties of a heteroskedastic model over the homoskedastic variant. In these simulations, a hybrid model involving both agent error and private information as sources of uncertainty is estimated. As argued in the previous section, such a model can only be estimated with a heteroskedastic error structure. I then compare this model to a heteroskedastic agent error, heteroskedastic private information, and homoskedastic SP models.

[Table 1 is about here]

The equations below summarize the general structure of the three Monte Carlo analyses. Table 1 lists the parameters manipulated in the Monte Carlo analyses and summarizes the values they take in each set of simulations.

$$
\begin{aligned}
& U_{1}\left(Y_{1}\right)=\beta_{110}+\beta_{11} X_{11} \\
& U_{1}\left(Y_{2}\right)=\beta_{12} X_{12} \\
& U_{1}\left(Y_{3}\right)=\beta_{130}+\beta_{13} X_{13}+\beta_{13 a} X_{1}+\beta_{13 b} X_{c} \\
& U_{2}\left(Y_{2}\right)=0 \\
& U_{2}\left(Y_{3}\right)=\beta_{230}+\beta_{23} X_{23}+\beta_{23 a} X_{2}+\beta_{23 b} X_{c}
\end{aligned}
$$

\footnotetext{
${ }^{12}$ Monte Carlo data sets and the simulation code are available upon request.
} 
where $X_{1}, X_{2}$, and $X_{c} \sim \operatorname{Uniform}(0,1)$, and the rest of $X_{i} \sim \operatorname{Normal}(0,1)$. For identification reasons, $U_{2}\left(Y_{2}\right)$ is normalized to zero and $U_{1}\left(Y_{2}\right)$ does not include a constant. There are several shared regressors in this specification: $X_{1}$ appears in Player 1's utility for outcome $Y_{3}$, as well as Player 1's private information variance specification. Similarly, $X_{2}$ appears in Player 2's utility for outcome $Y_{3}$ and Player 2's private information variance specification. $X_{c}$ is a common regressor that appears in both Player 1 and 2's utilities for $Y_{3}$, and also their private information variance specifications. The rest of the regressors are included in only one utility.

For the private information specification, which is used in Monte Carlo analyses 1 and 3, $\epsilon_{i j} \sim N\left(0, v_{i}^{2}\right)$, and

$$
\begin{aligned}
& v_{1}=\exp \left(\alpha_{1} X_{1 p}+\alpha_{1 a} X_{1}+\alpha_{1 b} X_{c}\right) \\
& v_{2}=\exp \left(\alpha_{2} X_{2 p}+\alpha_{2 a} X_{2}+\alpha_{2 b} X_{c}\right)
\end{aligned}
$$

where $X_{i p} \sim U(0,1)$, and the rest of the regressors are as defined previously. For the agent error variance, which is used in the data generating process of Monte Carlo experiment 3, $\varepsilon_{i j} \sim N\left(0, \nu_{i}^{2}\right)$, with

$$
\begin{aligned}
\nu_{1} & =\exp \left(\gamma_{1} X_{1 e}\right) \\
\nu_{2} & =\exp \left(\gamma_{2} X_{2 e}\right),
\end{aligned}
$$

where $X_{i e} \sim U(0,1)$.

\subsection{Analysis 1: Failure to Model Heteroskedasticity}

In this experiment, I evaluate the bias in a homoskedastic SP and an agent error HSP model when the data generating process is heteroskedastic with a private information component. The homoskedastic SP model used in the analysis employs a private information specifica- 
tion which does not correct for heteroskedasticity. The agent error heteroskedastic model corrects for heteroskedasticity, but specifies regressors $X_{i p}, X_{i}$, and $X_{c}$ for each player in the agent error variance parameter rather than the private information variance, resulting in a functional form misspecification. Finally, the private information HSP model follows the functional form and regressor specification of the data generating process.

The first row of Table 1 summarizes the parameter values used in the experiment. I generate simulation data based on these values, and estimate the three models for sample sizes $N=\{200,300,500,1000,2500,5000,10000\} .^{13}$

[Table 2 is about here]

Table 2 presents the estimation results for four coefficients from the models: $\beta_{13}, \beta_{23 b}, \alpha_{1 b}$, and the standard error estimate for $\beta_{13}$. For each sample size and the estimator, the table gives a $95 \%$ confidence interval based on the simulated coefficient values. In each cell, the top number is the lower bound, and the bottom value is the upper bound of the confidence interval. The table shows that, out of the three estimators considered, the private information HSP offers coefficients closest to the true data generating value for each parameter.

[Table 3 is about here]

However, a direct comparison of coefficients across the three models might potentially be misleading, as each coefficient might be scaled differently for each estimator due to the presence of heteroskedasticity. For this reason, Table 3 offers a more accurate comparison by evaluating each estimator's performance in predicting the probability of outcome 3, or $P\left(Y_{3}\right)=p_{1} \times p_{2}$, which is on average around .25 in the data generating process. For each

\footnotetext{
${ }^{13}$ For the first five sample sizes, 2500 iterations were completed. The number of Monte Carlo iterations is 2000 for $\mathrm{N}=5000$, and 750 for $\mathrm{N}=10000$.
} 
sample size, Table 3 offers three indicators:

$$
\begin{aligned}
\text { Mean Bias } & =\sqrt{\operatorname{mean}\left[\left(\operatorname{Pr}_{\mathrm{i}}^{\mathrm{E}}\left(\mathrm{Y}_{3}\right)-\operatorname{Pr}_{\mathrm{i}}^{\mathrm{DGP}}\left(\mathrm{Y}_{3}\right)\right)^{2}\right]} \\
\text { Maximum Bias } & =\sqrt{\max \left[\left(\operatorname{Pr}_{\mathrm{i}}^{\mathrm{E}}\left(\mathrm{Y}_{3}\right)-\operatorname{Pr}_{\mathrm{i}}^{\mathrm{DGP}}\left(\mathrm{Y}_{3}\right)\right)^{2}\right]} \\
\text { Median \% Bias } & =\operatorname{median}\left[\sqrt{\left(\operatorname{Pr}_{\mathrm{i}}^{\mathrm{E}}\left(\mathrm{Y}_{3}\right)-\operatorname{Pr}_{\mathrm{i}}^{\mathrm{DGP}}\left(\mathrm{Y}_{3}\right)\right)^{2}} / \operatorname{Pr}_{\mathrm{i}}^{\mathrm{DGP}}\left(\mathrm{Y}_{3}\right)\right]
\end{aligned}
$$

For an observation $i, \operatorname{Pr}_{i}^{E}\left(Y_{3}\right)$ denotes a given estimator's probability estimate for outcome $Y_{3}$, and $\operatorname{Pr}_{i}^{D G P}\left(Y_{3}\right)$ is the same outcome probability according to the data generating process. Intuitively, Mean Bias is the root mean squared error (RMSE), or it measures the average bias - in absolute terms - in the outcome probability estimate of a given estimator for a given observation. Each reported value in the table is the mean value of this statistic in the $2500 \mathrm{MC}$ iterations completed for a specific sample size. The standard deviation of the statistic is also reported in parentheses below the mean value. For instance, the first cell on the left reports a value .092, which indicates that in 2500 samples simulated for sample size $\mathrm{N}=200$, on average there was .092 bias in the outcome probability estimate of the private information heteroskedastic model. Similarly, Maximum Bias reports the largest bias, in absolute magnitude, in the probability estimate in a given simulation sample. The reported value in the table takes the mean of these largest bias values over the 2500 samples simulated. Finally, Median \% Bias calculates the bias in absolute terms as a percentage of the actual probability estimate from the true data generating process, and takes the median value in a given sample. The table reports the mean of these median values for the simulation samples. Substantively, this measure differentiates a .10 bias in the probability parameter when the true probability from the data generating process is .20 versus .80 . Even though the absolute bias is the same in both cases, the bias might be regarded as more severe in the former than the latter relative to the true probability value.

According to the Mean Bias measure, the HSP estimator with the private information specification produces probability estimates with a relatively small bias in small samples, 
and this bias disappears as the sample size gets larger. The agent error specification has a slightly larger bias, which also gets smaller as the sample size increases. The homoskedastic $\mathrm{SP}$, on the other hand, on average has more biased probability estimates. The bias in the homoskedastic SP estimates does not disappear as the sample size increases, and for a given observation, the expected bias converges to around .118. This is quite large considering that the average value of the outcome probability $P\left(Y_{3}\right)$ in the data generating process is .25. This is also verified by the Median \% Bias indicator. Even in samples of larger sizes, the homoskedastic estimator produces probability estimates with biases that are on average around $35 \%$ of the actual outcome probability. The private information and the agent error heteroskedastic models, in contrast, produce smaller biases in percentage terms, which disappear as the sample size increases.

Finally, in terms of the Maximum Bias criterion, the homoskedastic estimator on average produces at least one probability estimate that differs from $P\left(Y_{3}\right)$ by as much as .60 in each sample. This large bias does not disappear in larger sample sizes. In contrast, the private information and the agent error heteroskedastic specifications produce milder maximum biases, which get smaller for larger sample sizes. For $\mathrm{N}=10000$, for instance, the largest deviation from $P\left(Y_{3}\right)$ in the sample is on average about .19 for both estimators.

To sum up, the Monte Carlo analysis shows that, when heteroskedasticity is present in the data generating process of a SP model, the estimator should correct for it to avoid potential bias. The results suggest that, for a simple game structure like the one considered here, it does not make much practical difference if the agent error or the private information specification is used to model heteroskedasticity. As long as heteroskedasticity is accounted for in the estimation, the small misspecification of the functional form with the agent error model is not of a major concern. On the other hand, when heteroskedasticity is present, the failure to correct for it can result in bias and misleading inference. ${ }^{14}$

\footnotetext{
${ }^{14}$ One potential caveat to this result is that HSP is a slightly more data intensive approach than the
} 


\subsection{Analysis 2: Fitting a Heteroskedastic Model When Errors}

Are Homoskedastic

What happens if heteroskedasticity is not present in the data generating process and the analyst fits a heteroskedastic model? Would this be a source of bias or inefficiency in the estimates? This Monte Carlo experiment assesses the effects of running a HSP model, when the underlying model is in fact homoskedastic with private information specification. The second row of Table 1 lists the parameter values used to generate the Monte Carlo data for analysis. As the table shows, all the coefficients in the agent error and private information variance components take the value of 0 to make the data generating process homoskedastic. The two heteroskedastic models in the experiment unnecessarily include variables $X_{i p}, X_{i a}$, and $X_{c}$ in their uncertainty variance specifications for players $i=1,2$.

[Table 4 is about here]

Table 4 summarizes the simulation results for coefficients $\beta_{13}, \beta_{23 b}, \alpha_{1 b}$, and the standard error estimate of $\beta_{13}$. Not surprisingly, except for a minor small sample bias, the homoskedastic SP successfully captures all the parameters. Moreover, the confidence intervals produced by the homoskedastic estimator narrow as the sample size increases, indicating consistency. The HSP estimator with private information specification also approximates the population coefficient values well. As in the homoskedastic case, there seems to be a minor small sample bias which disappears with larger sample sizes. The agent error heteroskedastic model seems to fare slightly worse than the private information version for Player 1's coefficients, due to the misspecification of the uncertainty structure. Yet, even this estimator provides homoskedastic variant due to the extra parameters being estimated in the model. For this reason, especially in smaller samples, users are advised to carefully evaluate convergence of their estimates by running their model with multiple starting values, as well as utilizing different optimization algorithms that are offered by statistical software. 
close enough approximation for coefficients. The main difference between the homoskedastic versus the heteroskedastic variants seems to be estimation uncertainty, in particular the width of the confidence intervals, and the size of the standard errors. Not surprisingly, the presence of irrelevant parameters in the heteroskedastic estimators decreases the efficiency of the estimated coefficients. Both heteroskedastic models produce wider confidence intervals, and larger standard errors than the homoskedastic model.

Interestingly, the coefficient estimates for the variance specification of the two heteroskedastic models reveal that both models estimate coefficients that are not statistically different from zero when there is no heteroskedasticity. This is true even for the coefficient of the regressor $X_{c}$, which appears in both players' heteroskedastic variance specifications, as well as in their utility specifications for outcome $Y_{3}$. The models managed to isolate the effect of this variable on the utilities by estimating $\beta_{i b}$ successfully, and reported no effect for the variance specification by estimating $\alpha_{i b}$ not distinguishable from 0 . This is reassuring, as it means that the heteroskedastic models are not likely to result in incorrect inference regarding actor-level variance in the amount of uncertainty, and they are not more likely than the homoskedastic model to cause Type I errors.

As in the previous Monte Carlo analysis, I look at three indicators, Mean, Maximum, and Median \% Bias, to evaluate the three models' relative performance in predicting $\mathrm{P}\left(Y_{3}\right)$. These indicators are presented in Table 5. All three indicators suggest that, the homoskedastic SP model has a small sample advantage over the heteroskedastic variants in terms of the accuracy of the probability predictions. When $\mathrm{N}=200$, on average, a given prediction from the homoskedastic model has about .07 bias, while the two heteroskedastic models on average miss the true probability value by .10. However, this advantage disappears as the sample size increases. For $\mathrm{N}=10000$, the difference in the mean bias between the homoskedastic and the HSP models considered is less than .01. 
[Table 5 is about here]

To sum up, this Monte Carlo analysis demonstrates that, statistically, modeling heteroskedasticity when it is absent in the data generating process is not as serious of a problem as failing to model heteroskedasticity when it is present. The former is mainly an efficiency problem, which disappears in larger samples, while the latter is a potential source of bias and inconsistency. ${ }^{15}$

\subsection{Analysis 3: A Hybrid Heteroskedastic Model}

In this Monte Carlo experiment, I evaluate the bias in the agent error and private information HSP models, as well as a homoskedastic SP model, when the underlying theoretical model is a hybrid model that incorporates both agent error and private information as sources of uncertainty. The underlying game structure is the same as in the previous two Monte Carlo analyses. The last row of Table 1 lists the parameter values used to generate the data with a hybrid uncertainty structure. This hybrid heteroskedastic model includes $X_{i p}$ in the private information variance, and $X_{i e}$ in agent error variance for each player. The heteroskedastic private information model, in contrast, includes both $X_{i p}$ and $X_{i e}$ in the private information variance for each player. Similarly, the agent error heteroskedastic model includes these variables in the agent error variance. Finally, the homoskedastic model puts these regressors in each player's utility for outcome $Y_{3}$, and assumes a private information specification with a constant variance.

[Table 6 is about here]

\footnotetext{
${ }^{15}$ That being said, the inefficiency-bias trade-off between HSP and the homoskedastic SP needs to be evaluated carefully in small samples. When the sample is small, users should at least consider reporting both the HSP and the homoskedastic SP results. If none of the coefficients in the error specification are significant in HSP, the homoskedastic SP should be preferred.
} 
Table 6 presents the coefficient estimates of $\beta_{13}, \beta_{23 b}, \alpha_{1 b}$ from the four estimators. Similarly, Table 7 provides a comparison of the predictive performance of the four models by focusing on outcome probability estimates. Not surprisingly, the results suggest that the hybrid model provides probability estimates with the least bias among the four models. In contrast, the homoskedastic SP produces coefficients with the largest outcome probability biases. For each of the four estimators, the bias in probability estimates becomes smaller, and the estimates get more efficient as the sample size increases. However, even with $N=10000$, there is a sizeable average bias in the homoskedastic model's probability estimates for a given observation $(\sim .08)$. The agent error and the private information heteroskedastic models, in contrast, produce smaller biases on average $(\sim .04)$.

[Table 7 is about here]

To sum up, this analysis shows that, a hybrid heteroskedastic model incorporating both types of uncertainty can be estimated if mutually exclusive regressors are used to model the private information and the agent error variances for each player. Second, even if the underlying data structure is a hybrid model, fitting a heteroskedastic model with an agent error or private information specification does not cause serious problems: as long as heteroskedasticity is accounted for, misspecifying the uncertainty structure only introduces minor bias as long as all the regressors for heteroskedasticity are included in the variance specification. This is reassuring, because it suggests that even if the underlying hybrid model does not have mutually exclusive regressors for the agent error and the private information variance - hence a hybrid model cannot be identified - fitting a slightly misspecified heteroskedastic model with only one source of uncertainty present but all the regressors included provides a close enough approximation. The failure to model heteroskedasticity when it is present, on the other hand, creates more serious problems and increases the danger of misleading inference. 


\section{Applications of Heteroskedastic Strategic Models}

The previous section has only considered the statistical benefits of incorporating heteroskedasticity in strategic models for more accurate inferences. Given that many statistical models in the discipline are probably only approximations of more complex functional relationships, and various forms of misspecifications might almost always be present in empirical analyses due to data issues or computational difficulties, the bias reduction achieved by incorporating heteroskedasticity in a strategic model by itself might be considered a limited contribution. Fortunately, HSP models studied in this paper have significant substantive value as well, as they make possible addressing many interesting research questions that have not been considered before due to the lack of appropriate methods. Below, I discuss two application areas for heteroskedastic models. First, I provide a replication of an existing study that uses a homoskedastic strategic probit model. I then discuss the potential extension of the model I develop in this paper to recent strategic models of signaling and belief updating. These examples show that, using this method, interesting research questions can be addressed on the amounts of uncertainty in a strategic interaction.

\subsection{Application I: An Analysis of Speculative Currency Crises}

In order to demonstrate the benefits of using the HSP estimator, in this section I replicate Leblang (2003)'s analysis of speculative currency attacks. ${ }^{16}$ Speculative attacks on currencies is one of the important areas of research in international political economy (Obstfeld, 1996; Morris and Shin, 1998; Leblang, 2003; Leblang and Satyanath, 2006). The main goal of this replication is not to show that Leblang's results are biased due to unmodeled heteroskedasticity. Instead, this section aims to show that, by modeling the variance parameter

\footnotetext{
${ }^{16}$ I pick Leblang's analysis for replication because, first, it is an influential article that focuses on the strategic aspect of speculative attacks; second, it uses a SP estimator to incorporate strategic interaction into empirical analysis; and third, the replication data set was kindly provided by the author.
} 
in the study of speculative attacks, interesting new research questions can be addressed and additional insights can be gained that can improve our understanding of this important phenomenon, and structure the future debate on this topic.

Why do speculative attacks on exchange rate pegs occur? Why do some governments allow their currency to devalue while others take costly measures to defend their currency? To answer these questions, Leblang employs a homoskedastic SP model. He theorizes that speculative attacks are a result of strategic interaction between speculators in currency markets and policy makers in governments. He argues that speculators attack national currencies based on their expectations about policymakers' policy response, which in turn depends on institutional, electoral, and partisan factors (Leblang, 2003).

The interaction between international financial markets (Market) and policymakers in a government (Government) starts with Market deciding between initiating a speculative attack or not. If Market initiates, Government can either defend or devalue its currency. The three outcomes of the game, therefore, are Status Quo, Devaluation, and Defense. Using a homoskedastic SP model, Leblang estimates Market's Devaluation and Defense utilities, and Government's Devaluation utility with constants. To model Market's Status Quo utility, Leblang uses Capital controls, Log(reserves/base money), Exchange rate overvaluation, Domestic credit growth, U.S. domestic interest rates, Debt service, Contagion, and Number of prior attacks. In Government's Defend utility, included variables are Unified government, Log(exports/GDP), Campaign and election period, Post-election period, Right government, Real interest rate, Capital controls, and Log(reserves/base money). Table 9 provides descriptions of the variables used in replicating Leblang's results. ${ }^{17}$

[Table 9 is about here]

A replication of Leblang's homoskedastic SP results is presented in the left half of Table

\footnotetext{
${ }^{17}$ For a more detailed description of these variables and their coding, see Leblang (2003), page 544.
} 
8. The homoskedastic SP model that Leblang uses assumes that the uncertainty variance is constant across observations in the sample. Leblang's data set consists of monthly observations of 90 developing economies from January 1985 to December 1998, a total of 7240 observations. Given the size and the range of this sample, heteroskedasticity constitutes a valid concern that might affect inferences. More importantly, scholars might be interested in finding out factors influencing the levels of uncertainty in this strategic interaction. For example, it would be interesting to test if there are any factors affecting the likelihood of sub-optimality in Market's choice of launching speculative currency attacks against different governments, which can be captured with a HSP model with agent error specification.

[Table 8 is about here]

Thus, in Table 8, I also present the results from a HSP model with agent error specification, which hypothesizes that Market's agent error variance, or speculators' tendency to make suboptimal choices, changes across observations as a function of certain political factors in the target country. ${ }^{18}$ More specifically, I test the hypothesis that Market's tendency to make suboptimal speculative attacks increases during campaign and election periods, representing an environment with additional political uncertainty. I also test if the regime type of the target country, measured by the country's Polity score (Marshall and Jaggers, 2007), has any effect on Market's agent error variance in deciding to launch a speculative attack or not. ${ }^{19}$

According to Table 8, substantively interesting results emerge from the heteroskedastic

\footnotetext{
${ }^{18}$ The possibility that speculators are not perfectly rational, fully informed agents has been acknowledged in the literature before (e.g. Calvo and Mendoza (2000)).

${ }^{19}$ For simplicity, I assume that the agent error in Government's response to these attacks is homoskedastic, because of the small number of observations in which governments actually get to choose. In the sample, there are only 88 cases of speculative attacks out of 7240 observations, and this rarety of crisis cases makes it difficult to fit a heteroskedastic model for Government's decision node as well. With the availability of new data with more speculative attacks, a fully heteroskedastic model can be estimated.
} 
model's variance estimates. ${ }^{20}$ Results indicate that the politically uncertain campaign and election periods do not have a significant effect on Market's tendency to make suboptimal choices in their decision to initiate a speculative attack or not. In contrast, regime type has a significant effect on Market's error variance. The positive significant coefficient of the Polity variable suggests that, when speculators face a potential target country that is more democratic, they are more likely to make a choice that is suboptimal in deciding to launch a speculative attack against the country's currency. Substantively, based on this result, the agent error variance of Market increases by $22 \%$ from an autocratic to a democratic target state (the full range of the Polity variable). This is an important result, because it suggests that existing studies on democracy's effect on the likelihood of speculative attacks provide only a partial picture of the relationship between regime type and speculative attacks. While the previous literature has produced mixed results on this relationship (Leblang and Satyanath, 2006, 2008), the application of HSP in this paper suggests that democracy makes speculators more likely to make a suboptimal choice in their decision, which might in turn influence their likelihood of launching a speculative attack against a democratic country.

Why do democratic countries make markets more likely to pick suboptimal choices? One possibility is that there is additional uncertainty associated with democratic governments' responses to a potential speculative attack, which increases the likelihood of a suboptimal decision by speculators. Although it has been argued that democratic systems are more transparent and should be associated with reduced policy uncertainty (Broz, 2002), scholars have also argued that a divided government in a democracy might increase the uncertainty markets have (Leblang and Satyanath, 2006). More generally, the existence of multiple groups in democracies that might influence policy choices can make it hard for markets

\footnotetext{
${ }^{20}$ The utility estimates between the two models, on the other hand, are quite similar. Only a couple of differences exist between the utility estimates of Leblang's homoskedastic and the alternative HSP models. In particular, Reserves in Government's utility becomes significant, and Prior Attacks in Market's utility loses its significance in the heteroskedastic model.
} 
to predict a potential response by democratic governments to a speculative attack. For instance, while Tsebelis (2002) argues that the existence of veto players might make policy change difficult in political systems, it is also possible that the multiplicity of veto groups in democracies, and bargaining among them over policy decisions might introduce additional uncertainty for markets. In less democratic countries, in contrast, there are relatively fewer groups that might influence the government's response to a speculative attack, which reduces the uncertainty markets have about the government's response, reducing the likelihood that they make a suboptimal choice in their decision to initiate a speculative attack or not.

Even though the HSP model provides a significant coefficient for Democracy, does it significantly fare better than Leblang's original model in terms of model fit? I use a likelihood ratio test suggested by Alvarez and Brehm (1995) in the context of heteroskedasticity to evaluate if a heteroskedastic strategic probit model is necessary over the homoskedastic probit estimated by Leblang. In this test, the "null" model is the homoskedastic SP model that restricts the variance to be constant across observations, and the alternative model is the heteroskedastic model that does not make such a restriction. The test compares the likelihoods of the two models, by calculating the likelihood ratio statistic $L R S=-2\left(L_{H o m}-\right.$ $\left.L_{H e t}\right)$, where $L_{H o m}$ and $L_{H e t}$ are the log-likelihoods of the two models. This statistic follows a $\chi^{2}$ distribution, with degrees of freedom equal to the number of parameters in the variance specification of the heteroskedastic model. In comparing the two models in Table 8, LRS is equal to 6.17 , which is significant at the .05 level. This indicates that the heteroskedastic model provides a statistically significant improvement in model fit over Leblang's original results. ${ }^{21}$

\footnotetext{
${ }^{21}$ In a working paper, Keele and Park (2005) warn users of heteroskedastic probit models of the danger of fragile identification, which, they claim, can be hard to detect. As a minimum precautionary step, they suggest the users of heteroskedastic probit models to use different starting values in estimation. In this replication, I accordingly used 2,000 random starting values and a variety of maximization algorithms to estimate the model. At the end, estimation always reached the same set of coefficient values presented in Table 8 .
} 
Are there alternative mechanisms through which democracies might influence the agent error variance of markets? Are there other political or economic factors that influence markets' or governments' behavior and the overall amount of uncertainty in this strategic interaction? Further study beyond the scope of this paper is needed to answer these questions. The main goal of this replication was to show that modeling variance can reveal new and important information about the levels of uncertainty during speculative crises, which was not available from previous studies on this subject that mainly focus on explaining the likelihood of speculative attacks. Hopefully these results will initiate a fruitful debate on this topic.

\subsection{Application II: Heteroskedasticity and Bayesian Updating}

In recent years, increased attention has been given in the international relations literature to strategic models that incorporate signaling and Bayesian updating (Lewis and Schultz, 2003; Whang, 2010). The strategic models I have considered in this paper so far do not have this feature, as they involve only one decision node per actor in the game, making signaling and updating irrelevant. When an actor moves more than once in a game with uncertainty, however, the possibility of signaling and belief updating by players emerges, as players can come up with better estimates of their opponent's future behavior by observing their previous choices. Heteroskedasticity can easily be incorporated into these models as well, by linking the uncertainty parameter to regressors. Below, I discuss the potential statistical and substantive advantages of doing so.

[Figure 2 about here]

Figure 2 depicts a simple crisis model with signaling, analyzed in Lewis \& Schultz (2003). Two states, A and B, dispute over a good that is possessed by B. In this interaction, A first 
decides to challenge the status quo or not. If A does not challenge, status quo (SQ) is the outcome, with payoffs $S_{A}$ and $S_{B}$ to A and B, respectively. If A challenges B, B can resist the challenge, or concede the good. If B does not resist, the outcome CD gives the good to A peacefully, with payoff $V_{A}$ to $\mathrm{A}$, and $C_{B}$ to $\mathrm{B}$, representing the cost to $\mathrm{B}$ of losing the good. If B resists, on the other hand, A can either stand firm and use force (SF), or back down (BD). SF gives war payoffs $W_{A}$ and $W_{B}$ to $\mathrm{A}$ and $\mathrm{B}$, respectively, and $\mathrm{BD}$ gives the audience cost $a$ to $\mathrm{A}$, and $V_{B}$ to $\mathrm{B}$, representing the value of the good for $\mathrm{B}$.

This is a game of two-sided incomplete information where both states have uncertainty about their opponent's preferences. In particular, A is uncertain about B's payoff from war, $W_{B}$, and B is uncertain about both $a$ and $W_{A}$. Similar to the SP specification in the previous section, the uncertainty about the opponent's payoffs is represented by normally distributed random variables attached to observable outcome utilities: $W_{A}=\bar{W}_{A}+\epsilon_{A} ; a=\bar{a}+\epsilon_{a}$; and $W_{B}=\bar{W}_{B}+\epsilon_{B}$, where $\epsilon_{i}$ represent a player's private information that cannot be observed by the opponent.

There is signaling and updating in this game because A's first decision potentially reveals information about A's choice in this player's second decision node. In particular, B updates its belief about A's likelihood of fighting, after observing A's challenge decision. Lewis and Schultz (2003) use the Perfect Bayesian Equilibrium (PBE) concept to analyze the game and derive the equilibrium probabilities, including B's posterior and prior beliefs. The probabilities of A challenging $\left(p_{C}\right)$, B resisting $\left(p_{R}\right)$, B's prior belief about A fighting $\left(p_{F}\right)$, and B's posterior belief about A fighting given a challenge $\left(p_{F \mid C}\right)$ can be calculated as 
follows: ${ }^{22}$

$$
\begin{aligned}
p_{R} & =\Phi\left[\frac{p_{F \mid C}+\left(1-p_{F \mid C}\right) V_{B}-C_{B}}{p_{F \mid C} \sigma}\right] \\
p_{C} & =1-\Phi\left(\frac{S_{A}-\left(1-p_{R}\right) V_{A}-p_{R} \bar{W}_{A}}{p_{R} \sigma}\right) \Phi\left(\frac{S_{A}-\left(1-p_{R}\right) V_{A}-p_{R} \bar{a}}{p_{R} \sigma}\right) \\
p_{F \mid C} & =\Phi_{2}\left(\frac{\bar{W}_{A}-\bar{a}}{\sigma \sqrt{2}}, \frac{p_{R} \bar{W}_{A}+\left(1-p_{R}\right) V_{A}-S_{A}}{p_{R} \sigma}, \frac{1}{\sqrt{2}}\right) / p_{C} \\
p_{F} & =\Phi\left(\frac{\bar{W}_{A}-\bar{a}}{\sigma \sqrt{2}}\right)
\end{aligned}
$$

From a statistical point of view, this updating model has a relatively more complex game structure compared to the models I considered so far. Simple strategic models with no updating, as discussed in the previous section, result in a recursive system of probability equations, which implies dependence of actor choices on predictions about other actors' preferences later in the game. Hence, in the models I considered, any bias in a choice probability is transferred to the probability estimates for upper decision nodes, but this bias does not affect probability estimates for choices further down the game tree. The Bayesian updating model analyzed in Lewis \& Schultz (2003), in contrast, displays a stronger, nonrecursive interdependence of choices: because of the updating and signaling dynamics in the model, each actor choice probability depends directly or indirectly on the rest of the probabilities in the model, and any bias in any choice probability has implications for the rest of the probabilities.

Lewis \& Schultz (2003) make a homoskedasticity assumption about $\sigma$, the standard deviation of the random private information components capturing the amount of private information. Is this a restrictive assumption in a Bayesian updating model? It is clear that $\sigma$ plays a role in all four probabilities of Lewis \& Schultz's (2003) model. Thus, as in the simpler strategic models considered in the previous section, the misspecification of this parameter will likely be a source of bias in the equilibrium probability estimates. Moreover, because of

\footnotetext{
${ }^{22}$ A detailed derivation of these probabilities appears in Lewis \& Schultz (2003).
} 
the stronger interdependence in a signaling model, the failure to model heteroskedasticity if it exists - is likely to have even more serious effects on the estimation results and inferences than in a simple strategic model with no updating. ${ }^{23}$ This is especially a relevant issue in international relations empirical analysis as the data sets analyzed tend to be cross-sectional and show a high level of actor heterogeneity.

By affecting B's prior and posterior estimates of A's fighting choice, parameter $\sigma$ also indirectly affects the amount of updating by B in the Lewis \& Schultz (2003) model. The equilibrium amount of updating - the difference between B's prior and posterior estimates of Player A's probability of fighting at the last node - equals to:

$$
p_{F \mid C}-p_{F}=\frac{\Phi_{2}\left(\frac{\bar{W}_{A}-\bar{a}}{\sigma \sqrt{2}}, \frac{p_{R} \bar{W}_{A}+\left(1-p_{R}\right) V_{A}-S_{A}}{p_{R} \sigma}, \frac{1}{\sqrt{2}}\right)}{p_{C}}-\Phi\left(\frac{\bar{W}_{A}-\bar{a}}{\sigma \sqrt{2}}\right)
$$

In this expression, the variance parameter appears in a non-linear form in both B's posterior and prior estimates. Figure 3 plots B's prior and posterior probability estimates of A's fighting choice, and shows the amount of updating as a function of $\sigma$ for an example specification used in Lewis \& Schultz (2006). ${ }^{24}$ This example clearly shows that the amount of updating varies depending on the amount of initial uncertainty, and that it is non-linear and non-monotonic in the amount of private information Players A and B have. For this specific example, the largest amount of updating occurs when $\sigma \sim .83$, and the amount of updating diminishes at a higher rate for $\sigma<.83$ and at a lower rate for $\sigma>.83$.

[Figure 3 about here]

This relationship between $\sigma$ and updating is relevant to a recent debate in the literature regarding the amount of updating in strategic models of signaling. Scholars have argued

\footnotetext{
${ }^{23}$ Heteroskedasticity can easily be incorporated into this model by using the following link function: $\sigma=$ $\exp (\gamma Z)$ where $Z$ is the set of regressors (excluding a constant) that are expected to affect the error variance, and $\gamma$ is the coefficient vector for these regressors.

${ }^{24}$ In this example, $S_{A}=0, V_{A}=1, V_{B}=1, C_{B}=0, \bar{W}_{A}=-2.9, \bar{W}_{B}=-.5$, and $\bar{a}=-3.5$.
} 
that, in the existing statistical models of signaling, the estimated amount of updating by players remains limited (Lewis and Schultz, 2006; Wand, 2006). The analysis here suggests that misspecification of the uncertainty parameter is a potential reason for limited updating found in a signaling model that assumes homoskedastic errors. If a sample of international crises includes countries with different amounts of private information, which implies different levels of updating by their opponents, using a model that forces each country to have the same amount of private information might result in misleading inferences about not only actor choices but also the levels of updating by actors.

Modeling $\sigma$ with regressors to capture this heterogeneity, instead of assuming a constant $\sigma$ across all observations in the sample, can eliminate this bias, and also provide scholars with new insights into factors influencing the amount of private information and belief updating in international relations. In the international conflict literature, for instance, it has been argued that democracies signal their preferences more effectively during international crises. Modeling $\sigma$ can help us test if democracies enter into a crisis with lower levels of private information to begin with, or if their opponents experience a larger shift in their beliefs compared to opponents of other countries.

\section{Conclusion}

The objective of this paper has been to offer a method to capture actor-level variations in the amounts of uncertainty in statistical models of strategic interaction that has been gaining popularity in recent years in the international relations literature. The Monte Carlo analyses presented in this paper have shown that, if not controlled for, the presence of heteroskedasticity in the uncertainty parameters of such models can be a source of bias and inconsistency in estimates. The method proposed here accounts for heteroskedasticity, 
without adding significant complexity to the estimation and interpretation of these models.

More importantly, one major advantage of the proposed method is that it makes possible answering many substantively interesting questions about the levels of uncertainty in a strategic interaction, which have been mostly avoided in the literature due to the lack of appropriate methodological tools to measure the theoretical concepts. The models presented in this paper are valuable tools for analyzing factors that affect the severity of informational asymmetry, sources of suboptimal behavior, and the amount of Bayesian updating in strategic situations. They promise international relations researchers new and important venues for future research. 


\section{References}

Alvarez, Michael and John Brehm. 1995. "American Ambivalence Towards Abortion Policy: Development of a Heteroskedastic Probit Model of Competing Values." American Journal of Political Science 39:1055-82.

Alvarez, Michael and John Brehm. 2002. Hard Choices, Easy Answers. Princeton, New Jersey: Princeton University Press.

Bas, Muhammet A., Curtis S. Signorino and Robert W. Walker. 2008. "Statistical Backwards Induction: A Simple Method for Estimating Recursive Strategic Models." Political Analysis 16(1):21-40.

Braumoeller, Bear. 2006. "Explaining Variance; Or, Stuck in a Moment We Cant Get Out Of." Political Analysis 14:268-290.

Broz, Lawrence. 2002. "Political System Transparency and Monetary Commitment Regimes." International Organization 56:861-887.

Bueno de Mesquita, Bruce and David Lalman. 1992. War and Reason: Domestic and International Imperatives. New Haven: Yale University Press.

Calvo, Guillermo and Enrique Mendoza. 2000. "Capital-Markets Crises and Economic Collapse in Emerging Markets: An Information-Frictions Approach." American Economic Review 90:59-64.

Carrubba, Clifford, Amy Yuen and Cristopher Zorn. 2007a. "In Defense of Comparative Statics: Specifying Empirical Tests of Models of Strategic Interaction." Political Analysis 15(4):465-482.

Carrubba, Clifford, Amy Yuen and Cristopher Zorn. 2007b. "Reply to Signorino." Political Analysis 15(4):502-504.

Downs, George W. and David M. Rocke. 1979. "Interpreting Heteroskedasticity." American Journal of Political Science 23(4):816-828.

Esarey, Justin, Bumba Mukherjee and Will H. Moore. 2008. "Strategic Interaction and Interstate Crises: A Bayesian Quantal Response Estimator for Incomplete Information Games." Political Analysis 16:250-273.

Fearon, James D. 1994a. "Domestic Political Audiences and the Escalation of International Crises." American Political Science Review 88(3):577-92.

Fearon, James D. 1994b. "Signaling Versus the Balance of Power and Interests: An Empirical Test of A Crisis Bargaining Model." Journal of Conflict Resolution 38:236-269. 
Fearon, James D. 1995. "Rationalist Explanations for War." International Organization 49:379.

Greene, William H. 2003. Econometric Analysis. Upper Saddle River, NJ: Prentice Hall.

Harvey, A. 1976. "Estimating Regression Models with Multiplicative Heteroskedasticity." Econometrica 44:461-465.

Keele, Luke J. and David K. Park. 2005. "Ambivalent about Ambivalence: A Re-examination of Heteroskedastic Probit Models." Working Paper.

Leblang, David A. 2003. "To Defend or to Devalue: The Political Economy of Exchange Rate Policy." International Studies Quarterly 47:533-59.

Leblang, David A. and Shanker Satyanath. 2006. "Institutions, Expectations and Currency Crises." International Organization 60:254-262.

Leblang, David A. and Shanker Satyanath. 2008. "Journal of International Money and Finance." International Organization 27:480-497.

Lewis, Jeffrey and Kenneth Schultz. 2003. "Revealing Preferences: Empirical Estimation of a Crisis Bargaining Game with Incomplete Information." Political Analysis 11:345-367.

Lewis, Jeffrey and Kenneth Schultz. 2006. "Learning about Learning: a Response to Wand." Political Analysis 14:121-129.

Marshall, Monty G. and Keith Jaggers. 2007. "Polity IV Project: Political Regime Characteristics and Transitions, 1800-2006.".

McKelvey, Richard and Thomas Palfrey. 1995. "Quantal Response Equilibria for Normal Form Games." Games and Economic Behavior 10:6-38.

McKelvey, Richard and Thomas Palfrey. 1998. "Quantal Response Equilibria for Extensive Form Games." Experimental Economics 1:9-41.

McLean, Elena and Taehee Whang. 2010. "Friends or Foes? Major Trading Partners and the Success of Economic Sanctions." International Studies Quarterly 54:427-447.

Morris, Stephen and Hyun Song Shin. 1998. "Unique Equilibrium in a Model of Self-Fulfilling Currency Attacks." American Economic Review 88:587-597.

Obstfeld, Maurice. 1996. "Models of Currency Crisis with Self Fulfilling Features." European Economic Review 40:1037-47.

Ramsay, Kris and Curtis Signorino. 2009. "A Statistical Model of the Ultimatum Game." Working Paper. 
Schultz, Kenneth. 1998. "Domestic Opposition and Signaling in International Crises." American Political Science Review 92(4):829-44.

Schultz, Kenneth. 1999. "Do Domestic Institutions Constrain or Inform?: Contrasting Two Institutional Perspectives on Democracy and War." International Organization 53(3):233266.

Schultz, Kenneth. 2001. "Looking for Audience Costs." Journal of Conflict Resolution 45:3260 .

Signorino, Curtis S. 1999. "Strategic Interaction and the Statistical Analysis of International Conflict." American Political Science Review 93(2):279-97.

Signorino, Curtis S. 2003. "Structure and Uncertainty in Discrete Choice Models." Political Analysis 11:316-344.

Signorino, Curtis S. 2007. "On Formal Theory and Statistical Methods: A Response to Carrubba, Yuen, and Zorn." Political Analysis 15(4):483-501.

Signorino, Curtis S. and Ahmer R. Tarar. 2006. "A Unified Theory and Test of Extended Immediate Deterrence." American Journal of Political Science 50(3):586-605.

Signorino, Curtis S. and Kuzey Yilmaz. 2003. "Strategic Misspecification in Regression Models." American Journal of Political Science 47(3):551-66.

Smith, Alastair. 1999. "Testing Theories of Strategic Choice: The Example of Crisis Escalation." American Journal of Political Science 43(4):1254-1283.

Tsebelis, George. 2002. Veto Players: How Political Institutions Work. Princeton, New Jersey: Princeton University Press.

Wand, Jonathan. 2006. "Comparing Models of Strategic Choice: The Role of Uncertainty and Signaling." Political Analysis 14:101-120.

Whang, Taehee. 2010. "Empirical Implication of Signaling Models: Estimation of Belief Updating in International Crisis Bargaining." Political Analysis 18:381-402.

Yatchew, Adonis and Zvi Griliches. 1985. "Specification Error in Probit Models." The Review of Economics and Statistics 67(1):134-139. 


\section{A Appendix}

\section{A.1 Heteroskedastic SP with Private Information Specification}

With the private information specification, an important assumption in the underlying game theoretic model is that players possess private information about their payoffs, and as opposed to the agent error specification, they are assumed to act perfectly rationally given the information available to them. The true utility for Player $i$ from outcome $Y_{j}, j \in\{1,2,3\}$ is $\mathrm{U}_{\mathrm{i}}^{*}\left(\mathrm{Y}_{\mathrm{j}}\right)$, and only Player $i$ can observe this perfectly. The other player and the analyst have uncertainty about the exact payoff Player $i$ gets from outcome $Y_{j}$. The uncertainty is formalized as a stochastic term $\varepsilon_{\mathrm{ij}}$ attached to the outcome utilities:

$$
\mathrm{U}_{\mathrm{i}}^{*}\left(\mathrm{Y}_{\mathrm{j}}\right)=\mathrm{U}_{\mathrm{i}}\left(\mathrm{Y}_{\mathrm{j}}\right)+\varepsilon_{\mathrm{ij}}
$$

The systematic part of the utility $\mathrm{U}_{\mathrm{i}}^{*}\left(\mathrm{Y}_{\mathrm{j}}\right)$ is common knowledge to everyone, including the analyst. However, only Player $i$ observes $\varepsilon_{\mathrm{ij}}$ and the opponent and the analyst have distributional information about it. $\varepsilon_{\mathrm{ij}}$ are assumed to follow $\mathrm{N}\left(0, v_{\mathrm{ik}}^{2}\right)$.

Similar to the agent error specification, in order to derive the choice probabilities, we need to work backwards up the game tree. Player 2 chooses $a_{4}$ over $a_{3}$ if $U_{2}^{*}\left(a_{4}\right) \geq U_{2}^{*}\left(a_{3}\right)$. Thus,

$$
\begin{aligned}
p_{2}^{P I} & =\operatorname{Pr}\left(U_{2}^{*}\left(a_{4}\right) \geq U_{2}^{*}\left(a_{3}\right)\right) \\
& =\operatorname{Pr}\left(U_{2}\left(Y_{3}\right)+\varepsilon_{24} \geq U_{2}\left(Y_{2}\right)+\varepsilon_{23}\right) \\
& =\Phi\left(\frac{U_{2}\left(Y_{3}\right)-U_{2}\left(Y_{2}\right)}{\sqrt{v_{23}^{2}+v_{24}^{2}}}\right)
\end{aligned}
$$

Deriving Player 1's choice probabilities can be done in a similar way:

$$
\begin{aligned}
p_{1}^{P I} & =\operatorname{Pr}\left(\mathbb{E} \mathbb{U}_{1}\left(a_{2}\right) \geq \mathbb{E} \mathbb{U}_{1}\left(a_{1}\right)\right) \\
& =\operatorname{Pr}\left(\left(1-p_{2}^{P I}\right)\left(U_{1}^{*}\left(Y_{2}\right)\right)+p_{2}^{P I}\left(U_{1}^{*}\left(Y_{3}\right) \geq U_{1}^{*}\left(Y_{1}\right)\right)\right. \\
& =\operatorname{Pr}\left(\left(1-p_{2}^{P I}\right)\left(U_{1}\left(Y_{2}\right)+\varepsilon_{13}\right)+p_{2}^{P I}\left(U_{1}\left(Y_{3}\right)+\varepsilon_{14}\right) \geq U_{1}\left(Y_{1}\right)+\varepsilon_{11}\right) \\
& =\Phi\left(\frac{\left(1-p_{2}^{P I}\right) U_{1}\left(Y_{2}\right)+p_{2}^{P I} U_{1}\left(Y_{3}\right)-U_{1}\left(Y_{1}\right)}{\sqrt{v_{11}^{2}+\left(1-p_{2}^{P I}\right)^{2} v_{13}^{2}+p_{2}^{P I^{2}} v_{14}^{2}}}\right)
\end{aligned}
$$

$v_{\mathrm{ij}}^{2}$, variance terms of the stochastic $\varepsilon_{\mathrm{ij}}$, represent the severity of informational asymmetry. The larger $v_{\mathrm{ij}}^{2}$, the larger on average the private information Player $i$ possesses. $\varepsilon_{\mathrm{ij}}$ terms are distributed normally with zero mean and standard deviation $v_{\mathrm{ij}}$. As in the agent error specification, the standard deviation varies across different observations as functions of regressors:

$$
v_{\mathrm{ij}}=\exp (\lambda \mathbf{D})
$$


where $\lambda$ represents the coefficients and $\mathbf{D}$ is the matrix of regressors. ${ }^{25}$ The log-likelihood function for the HSP estimator for private information specification looks exactly the same as the agent error version, except the difference in outcome probability estimates, and is specified as follows:

$$
L^{P I}=\sum_{n=1}^{N} \sum_{j=1}^{3} I_{n j} \ln \left(\operatorname{Pr}^{P I}\left(Y_{n j}\right)\right)
$$

where $\operatorname{Pr}^{P I}\left(Y_{1}\right)=1-p_{1}^{P I}, \operatorname{Pr}^{P I}\left(Y_{2}\right)=p_{1}^{P I}\left(1-p_{2}^{P I}\right)$, and $\operatorname{Pr}^{P I}\left(Y_{3}\right)=p_{1}^{P I} p_{2}^{P I}$.

The expressions in equations 4 and 33 reveal that the agent error and private information specifications yield the same probability estimates for the second player. The difference is in action probabilities for decision nodes higher in the game tree, as can be seen from equations 7 and 37.

${ }^{25}$ As in the agent error case, the constant is omitted from this specification. 


\section{A.2 Sample STATA Code for a Heteroskedastic SP Model}

We can estimate a HSP model in STATA very easily. In order to do so, we first define our log-likelihood function, and then use the $\mathrm{ml}$ command available in Stata to perform the maximum likelihood estimation. Suppose that the data generating process comes from the game presented in Figure 1(A). The two variables that represent $P_{1}$ and $P_{2}$ 's choices are Attack, and Resist, respectively. In this example, we model utilities $U_{1}(C a p)$ with a regressor $X_{1}, U_{1}($ War $)$ with a regressor $X_{2}$ and a constant, and $U_{2}($ War $)$ with a regressor $X_{3}$ and a constant. In addition, $P_{1}$ and $P_{2}$ 's agent error variances $\alpha_{1}$ and $\alpha_{2}$ are modeled with $Z_{1}$ and $Z_{2}$, respectively. ${ }^{26}$

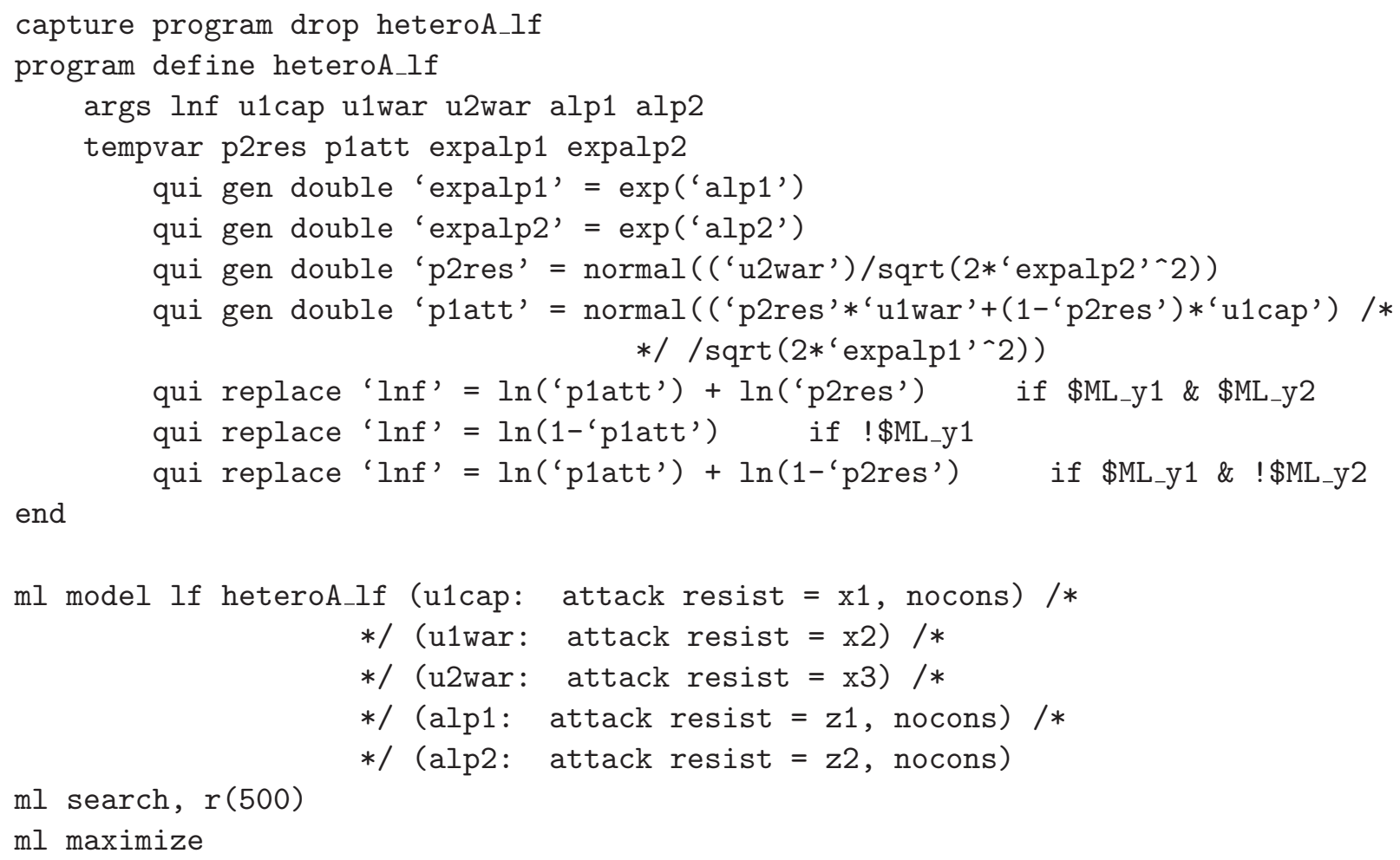

${ }^{26}$ In this example, $U_{1}(S Q)$ and $U_{2}(C a p)$ are normalized to zero. 


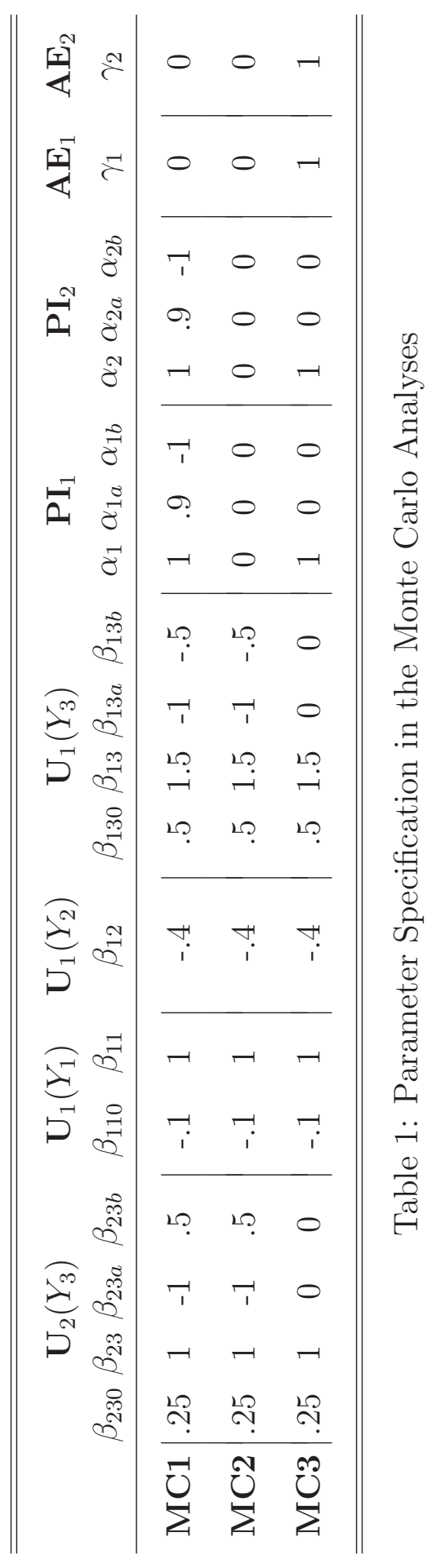




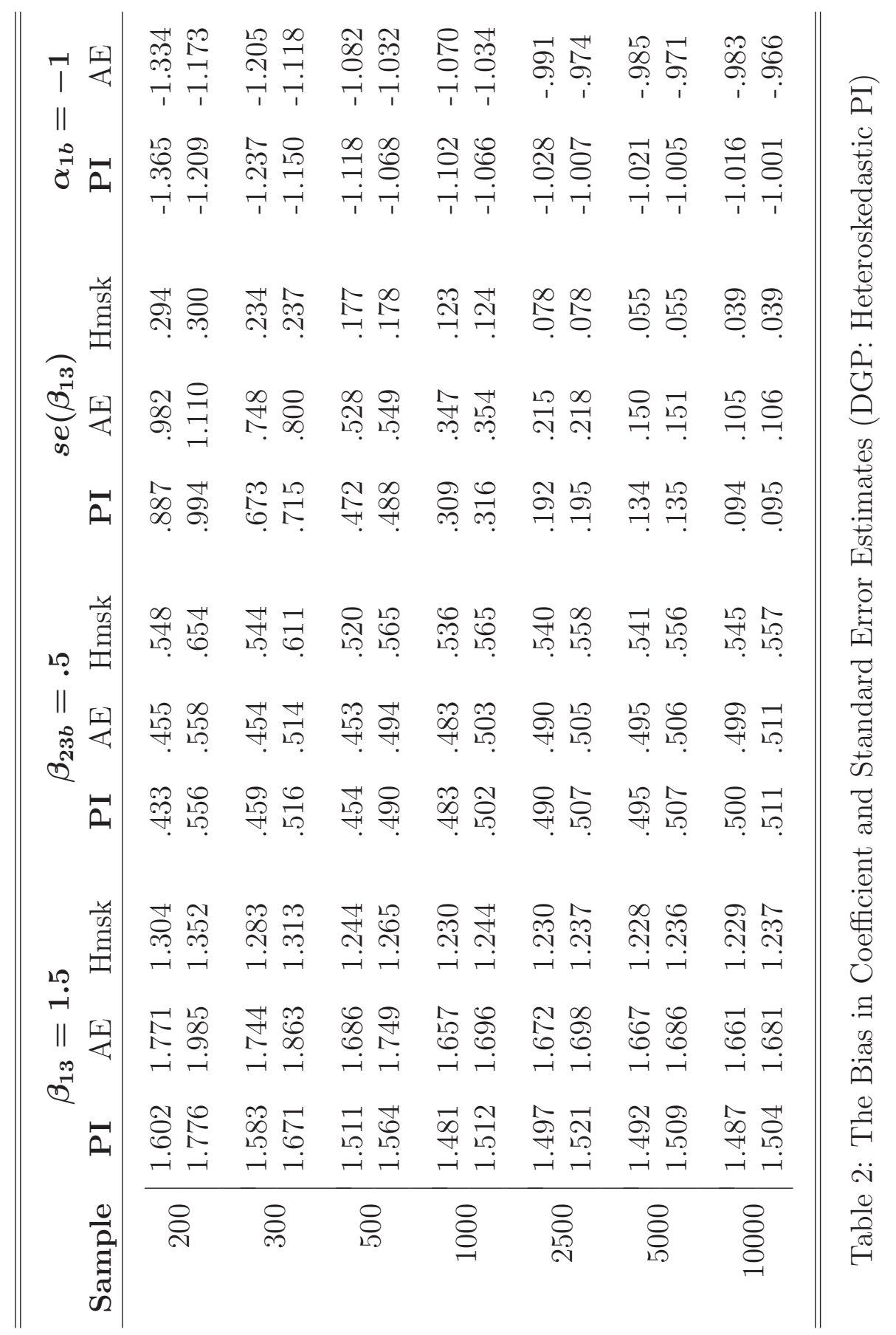




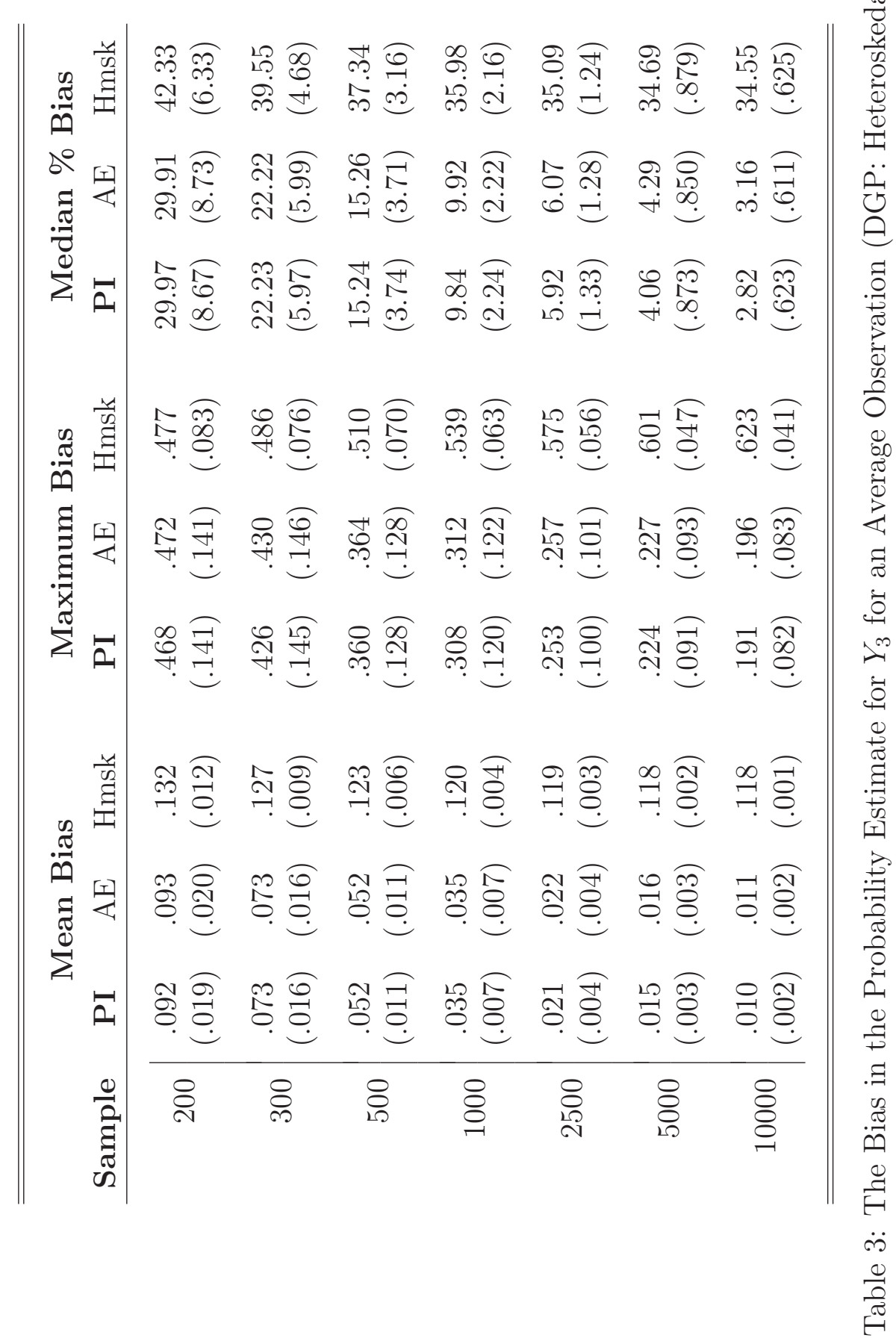




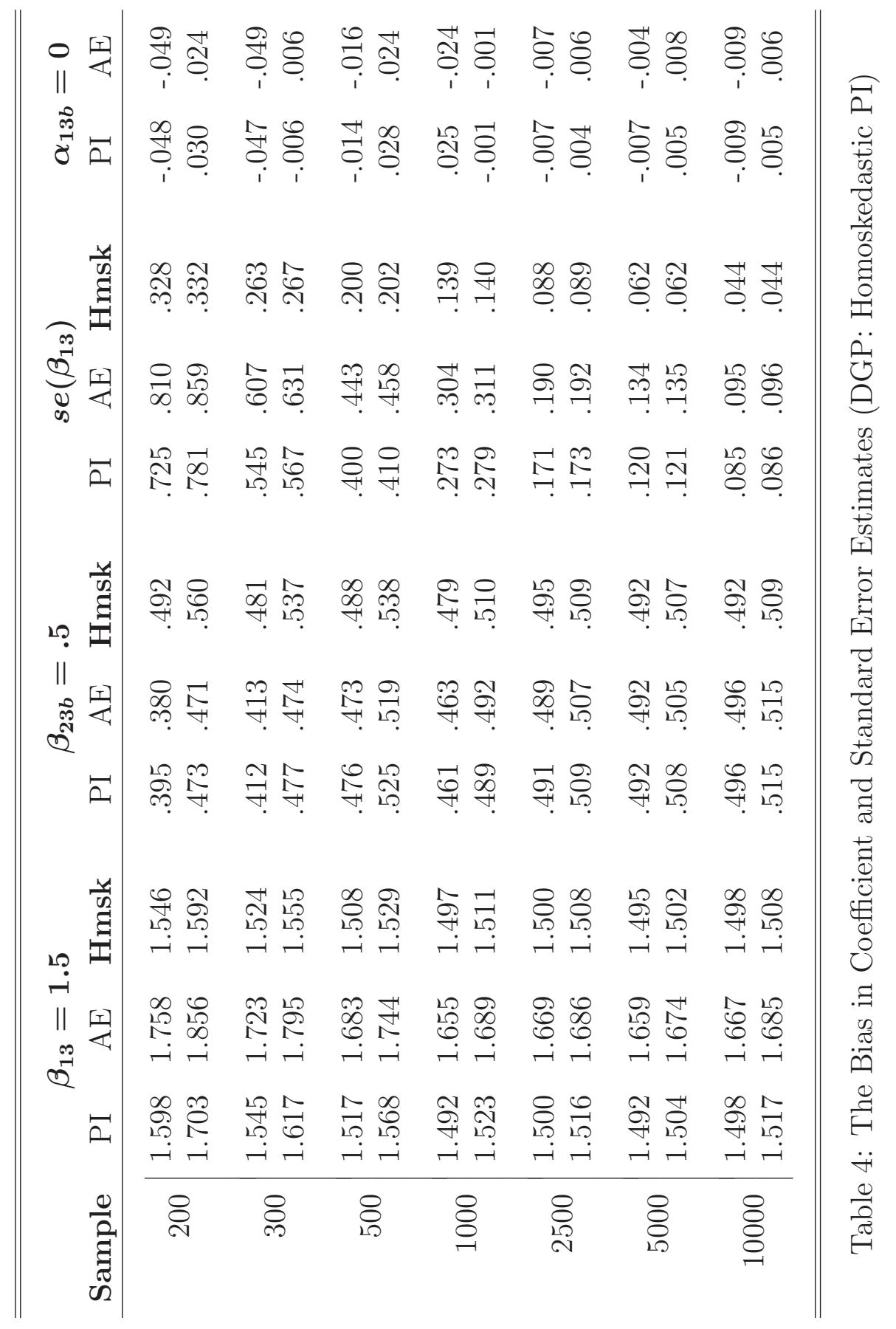




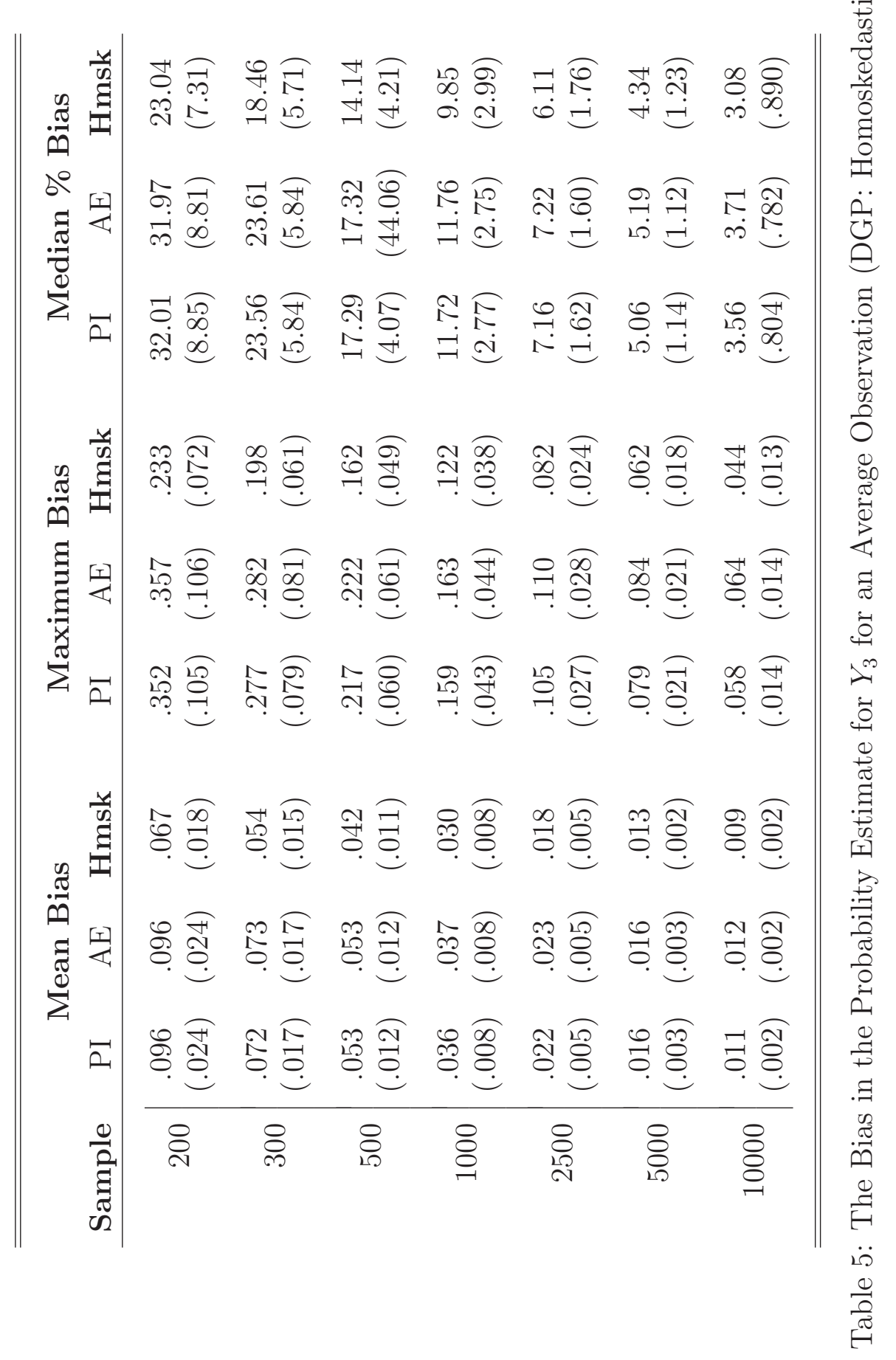




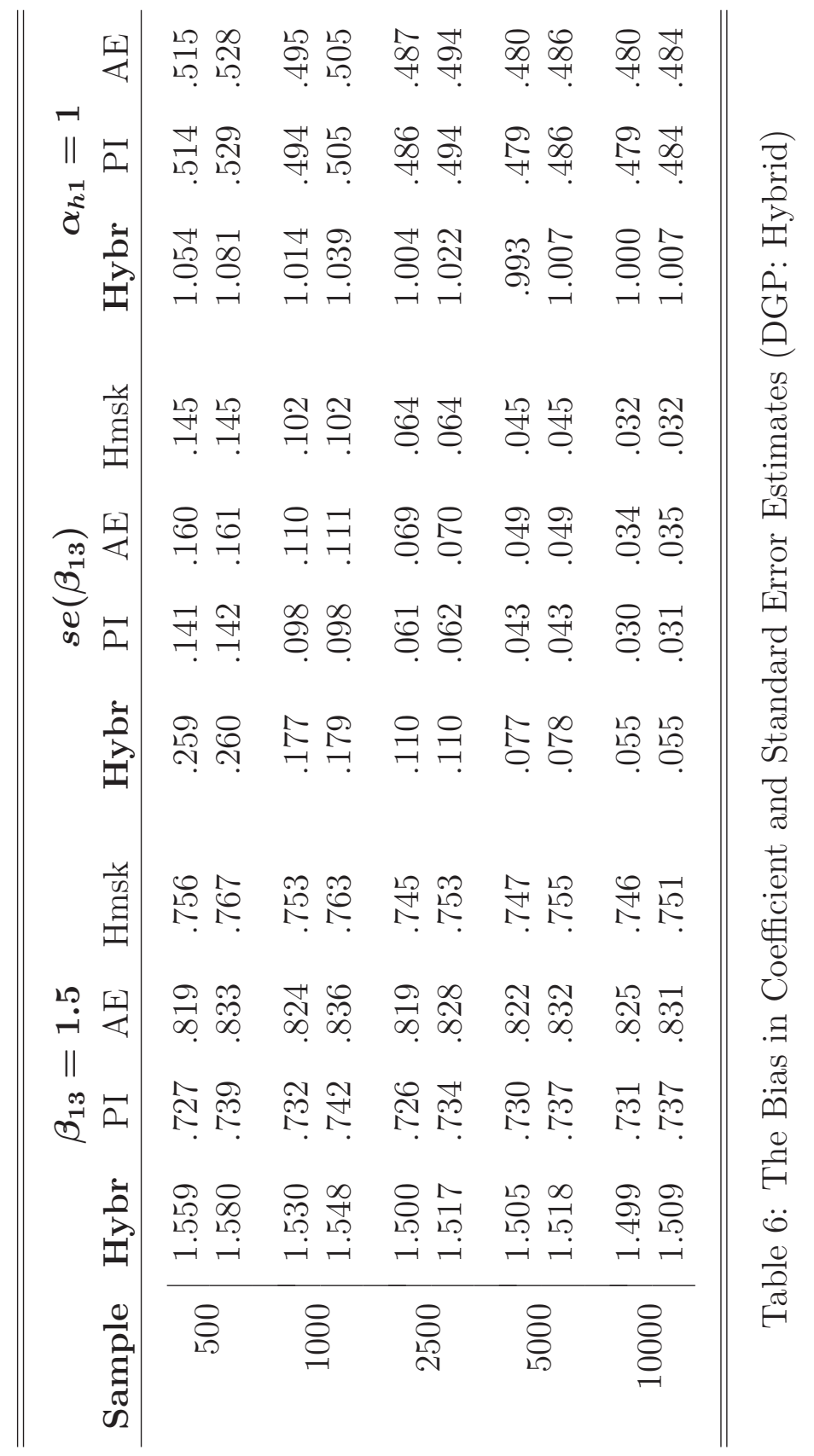




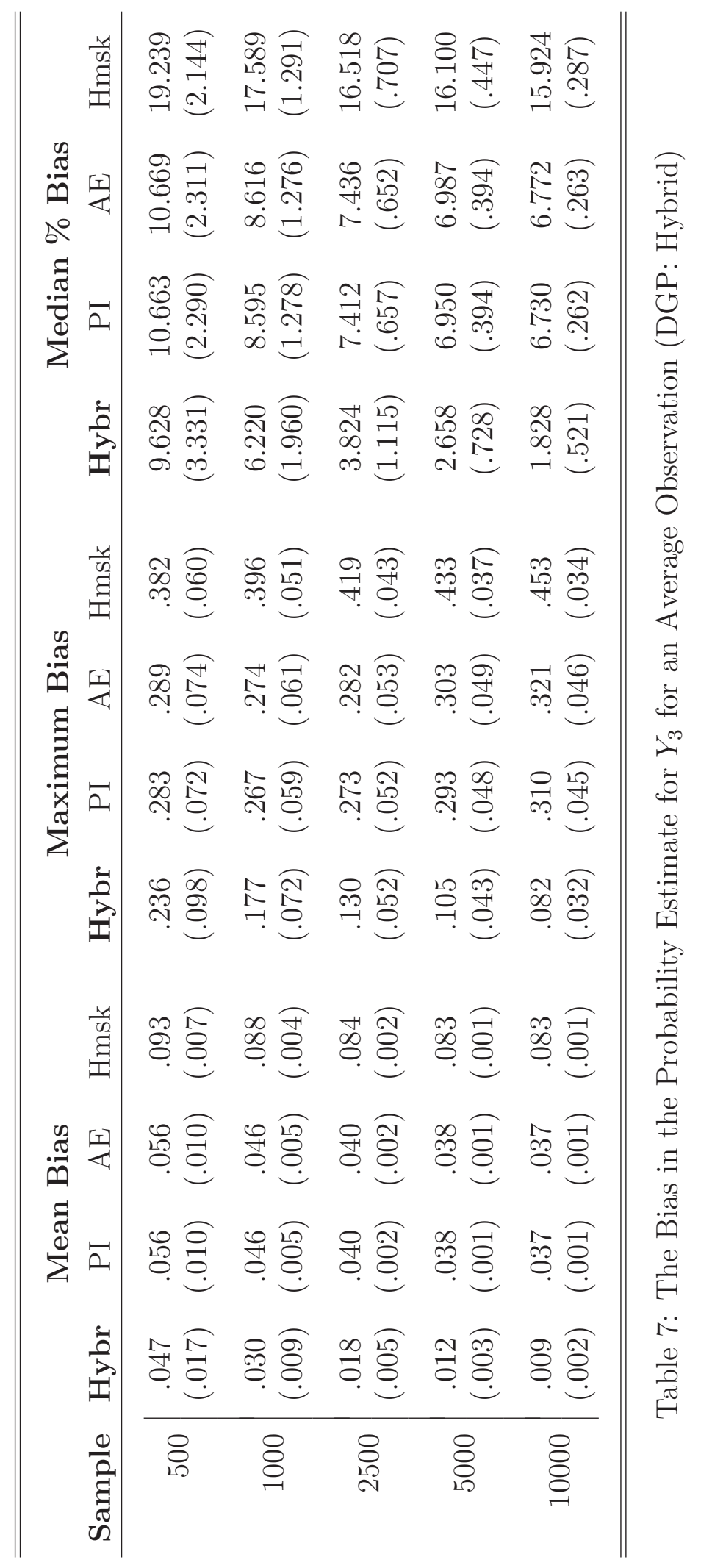




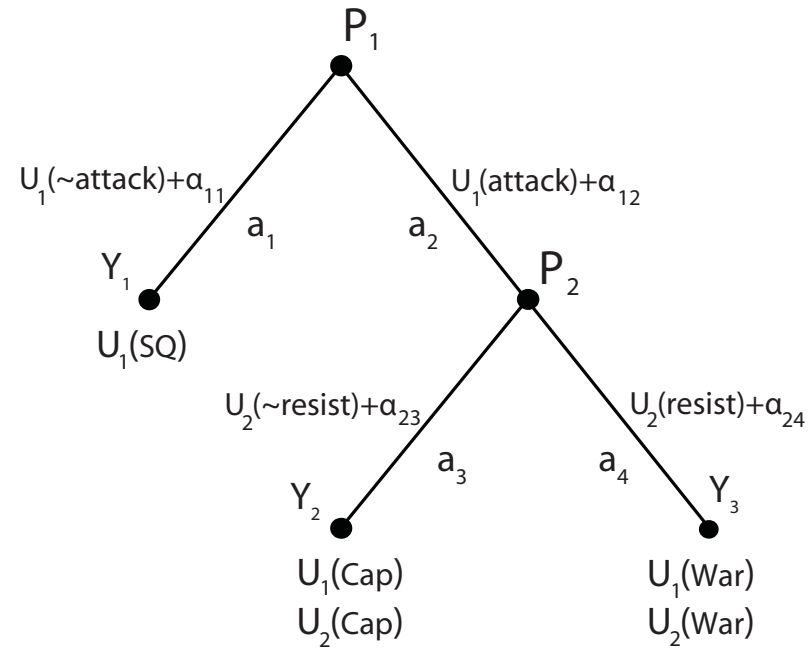

(A) Agent Error

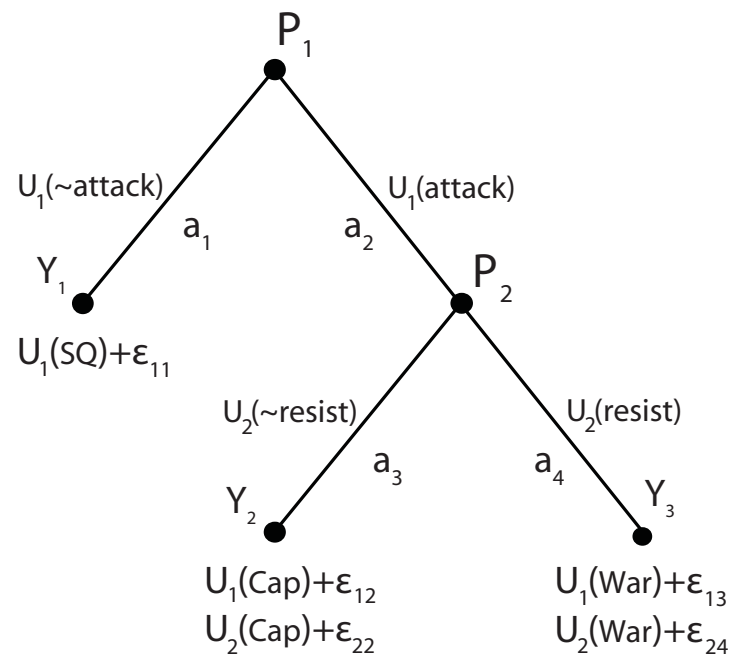

(B) Private Information about Payoffs

Figure 1: Theoretical Sources of Uncertainty in a Strategic Model 


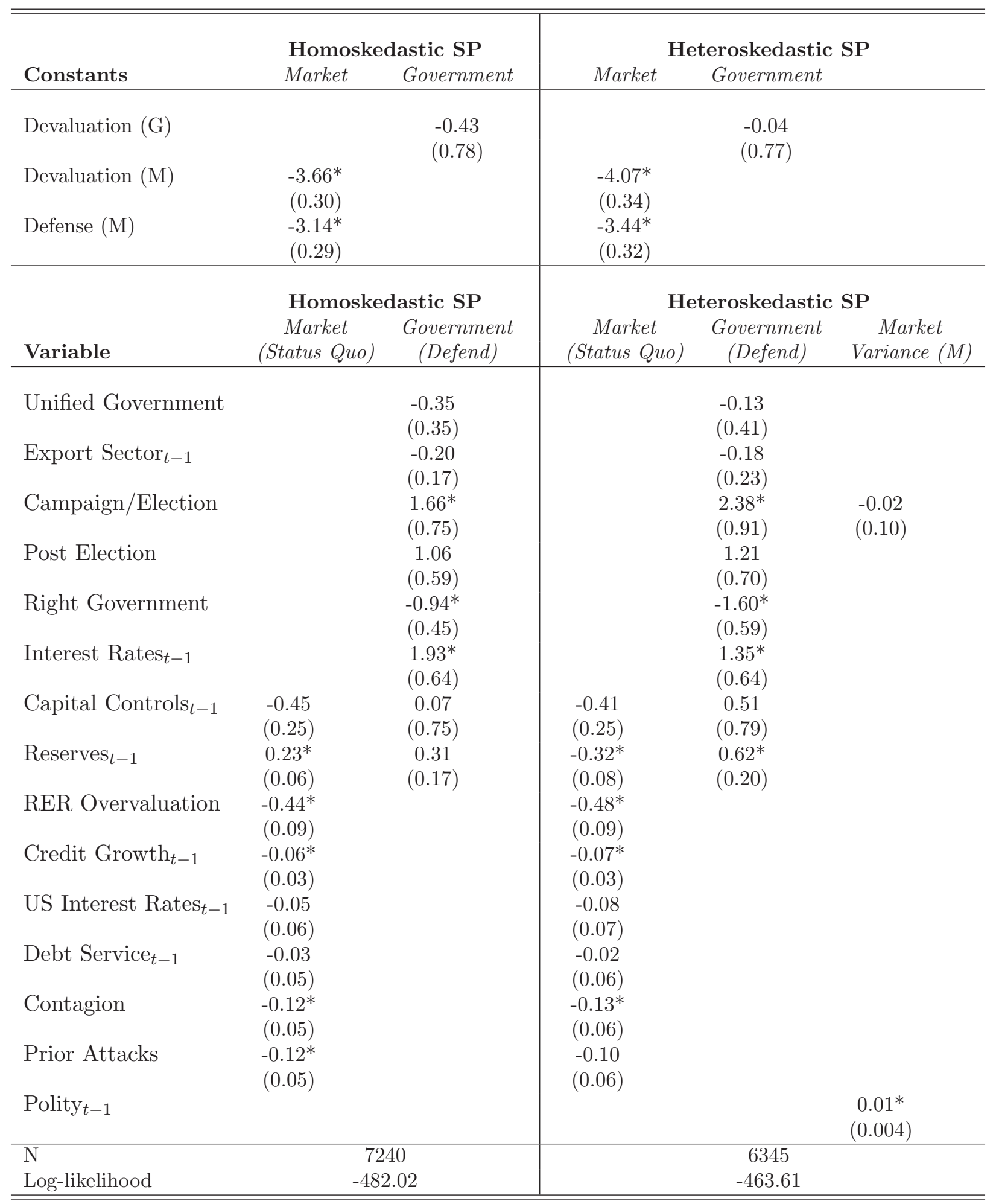

Table 8: Comparison of Leblang's Homoskedastic Model with an Alternative Heteroskedastic Model 


\begin{tabular}{|c|c|}
\hline Variable & Description \\
\hline Unified Government & $\begin{array}{l}\text { Binary indicator for the ruling party controlling } \\
\text { both the executive and the lower house of the legislature }\end{array}$ \\
\hline Export Sector St-1 $_{1}$ & Size of the export sector relative to GDP \\
\hline Campaign/Election & Binary indicator for campaign ( 3 months) and election times \\
\hline Post Election & $\begin{array}{l}\text { Binary indicator for the } 3 \text {-month period following } \\
\text { an election month }\end{array}$ \\
\hline Right Government & Binary indicator for a right government \\
\hline 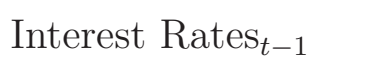 & Real interest rates \\
\hline Capital Controls $\mathrm{s}_{t-1}$ & Binary indicator for controls on the capital account \\
\hline Reserves $_{t-1}$ & Log-ratio of total reserves to base money \\
\hline RER Overvaluation & Real exchange rate overvaluation \\
\hline Credit Growth t-1 $_{t}$ & Domestic credit growth rate \\
\hline US Interest Rates $_{t-1}$ & U.S. domestic interest rates \\
\hline Debt Service $_{t-1}$ & External debt service \\
\hline Contagion & $\begin{array}{l}\text { Number of speculative attacks outside the country } \\
\text { in the same month }\end{array}$ \\
\hline Prior Attacks & Number of previous speculative attacks to the country \\
\hline Polity $_{t-1}$ & Polity score of the country \\
\hline
\end{tabular}

Table 9: Descriptions of the Variables Used in Leblang (2003) 


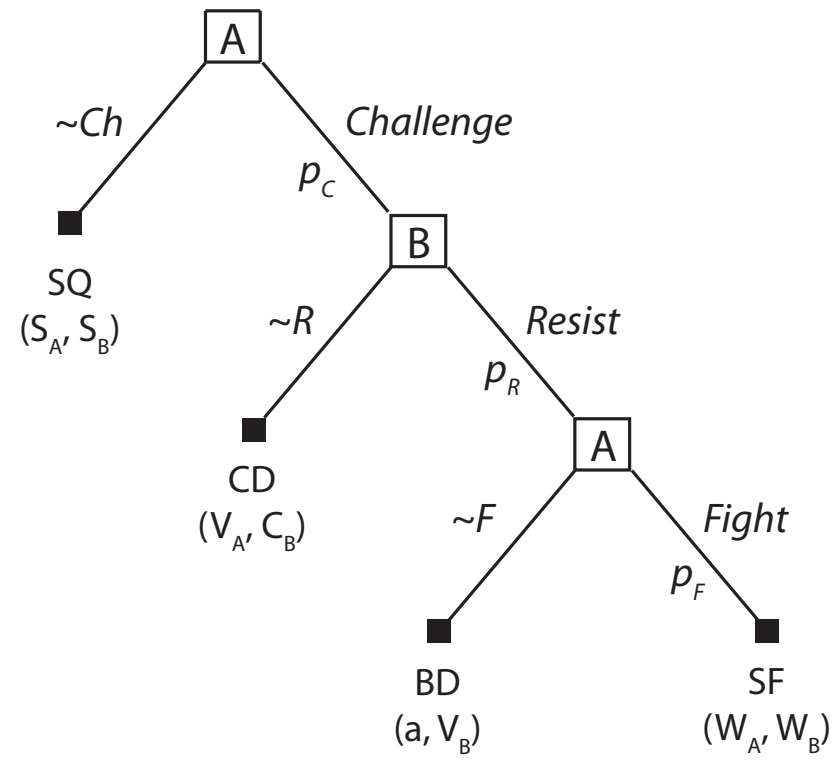

Figure 2: A Crisis Bargaining Game with Bayesian Updating 

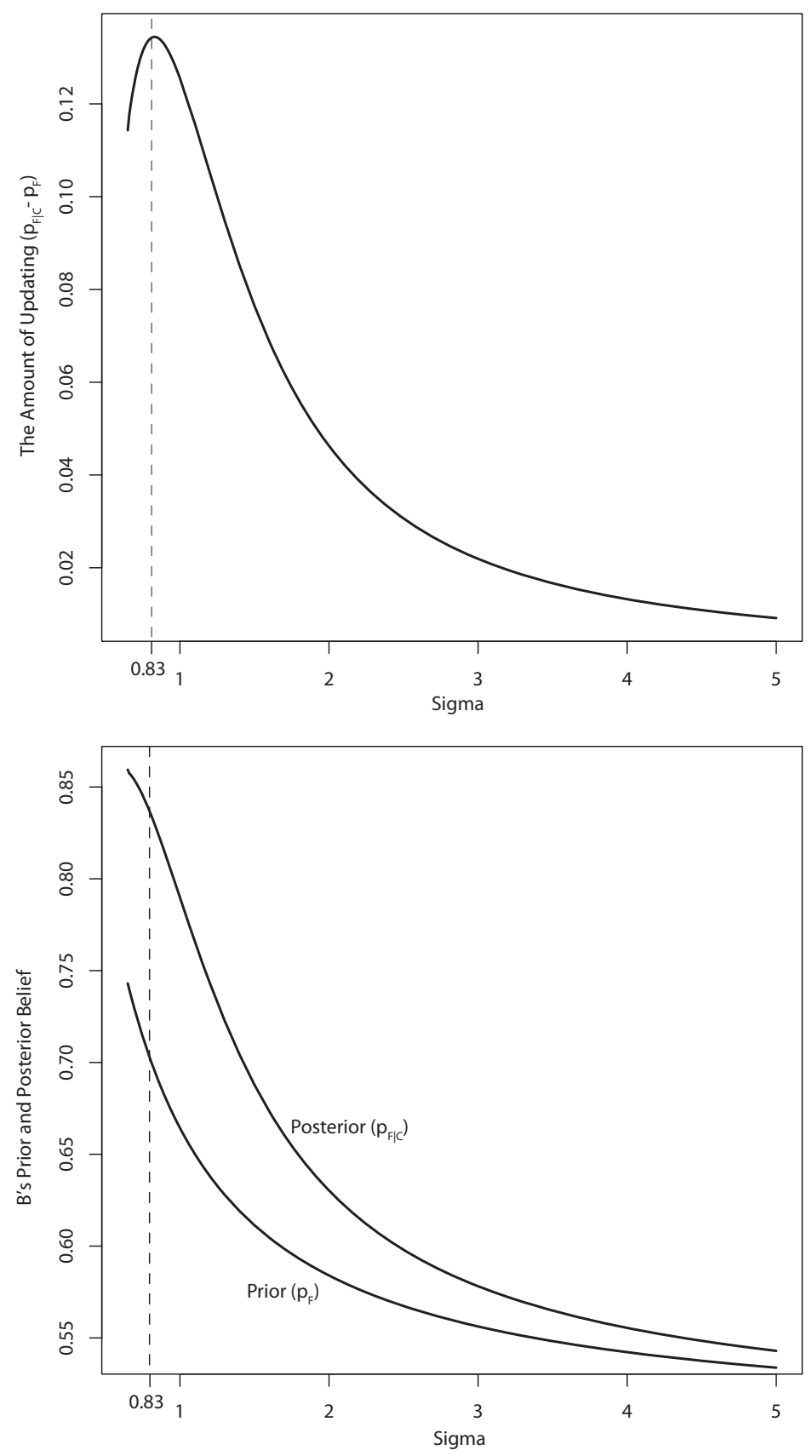

Figure 3: Sigma and the Amount of Updating 